Review

\title{
Fucoidans: Downstream Processes and Recent Applications
}

\author{
Ahmed Zayed ${ }^{1,2}$ (D) and Roland Ulber ${ }^{1, *}$ \\ 1 Institute of Bioprocess Engineering, Technical University of Kaiserslautern, Gottlieb-Daimler-Straße 49, \\ 67663 Kaiserslautern, Germany; ahmed.zayed1@pharm.tanta.edu.eg \\ 2 Department of Pharmacognosy, Tanta University, College of Pharmacy, El Guish Street, Tanta 31527, Egypt \\ * Correspondence: ulber@mv.uni-kl.de; Tel.: +49-0631-205-4043; Fax: +49-6312-05-4312
}

Received: 6 February 2020; Accepted: 15 March 2020; Published: 18 March 2020

\begin{abstract}
Fucoidans are multifunctional marine macromolecules that are subjected to numerous and various downstream processes during their production. These processes were considered the most important abiotic factors affecting fucoidan chemical skeletons, quality, physicochemical properties, biological properties and industrial applications. Since a universal protocol for fucoidans production has not been established yet, all the currently used processes were presented and justified. The current article complements our previous articles in the fucoidans field, provides an updated overview regarding the different downstream processes, including pre-treatment, extraction, purification and enzymatic modification processes, and shows the recent non-traditional applications of fucoidans in relation to their characters.
\end{abstract}

Keywords: fucoidans; extraction; brown algae; production; bioactivities

\section{Introduction}

Polysaccharides, nucleic acids, and peptides are considered the main three types of bioactive polymeric macromolecules [1]. Among these, polysaccharides serve various roles in living cells including structural functions, where cellulose and chitin represent the major components of the different cell wall matrices [2,3], energy storage (e.g., starch and glycogen) [4,5], and hydration and signaling functions (e.g., mucilage and alginic acid) [6,7].

Particularly, marine homo- and heteropolysaccharides are derived from marine organisms, which represent a large part of global biodiversity [8]. Among these are the algal polysaccharides, such as fucoidan and alginate in brown seaweeds, carrageenan in red seaweeds and ulvan in green seaweeds. These were reported to have interesting nutraceutical, biomedical, pharmaceutical and cosmeceutical applications, including dietary fibers; anti-inflammatory, anti-tumor, anti-oxidant, hepatoprotective and anti-coagulant properties; and drug carrier functionality. Therefore, they have been extensively investigated during the last few decades [9-13], especially after the emergence of glycobiology and glycomics [14-17].

Polysaccharides such as dietary fibers of brown algae are abundant and diverse (e.g., alginates, cellulose, fucoidans and laminarins) constituting the major components (up to 75\%) of the dried thallus weight (\% DW) [18-20]. Previous work investigated their abundance in different species, reporting Fucus, Ascophyllum, Saccharina, and Sargassum to contain 65.7, 69.6, 57.8 and 67.8 \% DW, respectively [21,22]. Specifically, fucoidans are found in the cell walls and extracellular matrices of brown algae in addition to more than 265 genera and 2040 species of marine invertebrates (e.g., sea cucumbers), where they perform vital structural functions [23-26]. Fucoidans are assumed to act as cross-linkers between the major threads of cellulose and hemicellulose, promoting cellular integrity and maintaining cellular hydration (especially during drought seasons) [27]. They also act in other 
reproductive, immune and cell-to-cell communicative roles [23]. As recommended by the International Union of Pure and Applied Chemistry (IUPAC), fucoidans is a general term used to describe sulfated L-fucose-based polymers including sulfated fucans cited by the Swedish scholar Kylin, as well as other fucose-rich sulfated heteropolysaccharides $[23,28]$. Their chemical structures, in terms of monomeric composition and branching, are quite simple in marine invertebrates compared to their analogues in brown algae [13,29].

Hundreds of articles have thoroughly discussed and reviewed the biological, pharmacological and pharmaceutical applications of fucoidans [30-33], including nanomedicine, [34] which has made it a hot topic in the last few decades [35-37]. All these studies tried to investigate fucoidans molecular mechanisms in relation to their chemical structure and physicochemical properties. Therefore, different hypotheses were suggested for each activity, such as anti-tumor [31,38-40], anti-coagulant [41,42], anti-viral $[43,44]$ and anti-inflammatory activity $[45,46]$. These investigations revealed that various factors are relevant, such as molecular weight, sulfation pattern, sulfate content and monomeric composition [47-49]. For example, different fractions were produced with different physicochemical properties in our previous experiments; sulfation pattern and sulfate content were highly related to anti-viral and cytotoxic activities against HSV-1 and Caco-2 cell lines, respectively, while molecular weight and sugar composition were potential factors in anti-coagulation activity [41,50]. In addition, degree of purity was reported as an influential factor [32], where co-extracted contaminants (e.g., phlorotannins or polyphenols) could lead to significant interference in anti-oxidant activity and, consequently, cosmetic applications [51,52].

Therefore, several key production challenges regarding fucoidans were discussed in our last review article in order to obtain a product that follows the universal good manufactured practice (GMP) guidelines. The article discussed sources of heterogeneity in extracted fucoidans, including the different biotic (e.g., biogenic, geographical and seasonal factors) and abiotic (e.g., downstream processes) factors affecting the fucoidans physicochemical and chemical properties [53]. Others patented production techniques that have assisted in the marketing of several commercial fucoidans by well-known companies (e.g., Sigma-Aldrich ${ }^{\circledR}$, Algues and Mer and Marinova ${ }^{\circledR}$ ) derived from Fucus vesiculosus and other brown algae species [54-56].

Furthermore, the improvement of fucoidans activity was investigated, targeting several points. Among these was the modification of the chemical structure of the native fucoidans scaffolding, including depolymerization [57,58] and over-sulfation [59]. These modifications could be attempted chemically [60], enzymatically [35,61] or physically [62]. Predetermined synthesis of oligomers [63,64] and low molecular weight polymers with defined monomeric units [65] is also involved. Additionally, fractionation of fucoidans is a common approach during extraction and purifications steps by applying different extraction and purification conditions (e.g., pH, time, molarity of $\mathrm{NaCl}$ [49,55].

The current article aimed at complementing our previously published article discussing the reasons for heterogeneity of fucoidans [53]. It reviewed and evaluated the different downstream processes used in production as the most important abiotic factors affecting the fucoidans quality and structural features; it then addressed recent uncommon applications and prospective bioproduction of fucoidans. In addition, the updated status of enzymatic structural modifications of fucoidans, especially by fucoidanases, were presented.

\section{Global Market and Cultivation of Brown Algae}

Marine hydrocolloids (e.g., agar, carrageenan and alginate) are of particular industrial interest, with worldwide annual production of approx. 100,000 tons and a value above US \$1.1 billion [66]. Based on FAO periodical reports (FAO, 2014, 2016), among the top seven most-cultivated seaweeds, three taxa are mainly used for hydrocolloids production; these include Rhodophyta (e.g., Eucheuma sp. and Kappaphycus alvarezii) for carrageenan production and Gracilaria sp. for agar production [67]. These data encouraged the global marine market to escalate the production yield by finding alternative, eco-friendly seaweed cultivation techniques, such as sea farming or aquaculture and biotechnology [53]. 
In 2014, the annual production of cultivated seaweeds reached 27.3 million tons [68], representing $27 \%$ of the total marine aquaculture production, while the global market of marine biotechnology (blue biotechnology) for industrial applications has been expected to achieve US \$4.8 billion in 2020 and grow to US $\$ 6.4$ billion by 2025 [69].

Species of brown macroalgae (Phaeophyceae) are distributed among the orders Fucales and Laminariales, which are the major commercial sources of the algal sulfated polysaccharides, in addition to Chordariales, Dictyotales, Dictyosiphonales, Ectocarpales, and Scytosiphonales. Moreover, phylogenetic analysis showed that Fucales are one of the largest and most diversified orders within Phaeophyceae, having eight families (41 genera and 485 species), named Ascoseiraceae, Cystoseiraceae, Durvillaeaceae, Fucaceae, Hormosiraceae, Himanthaliaceae, Sargassaceae, and Seirococcaceae [70]. Figure 1 illustrates the distribution of several examples of well-known brown algae species which are considered potential sources of sulfated polysaccharides dominating tropical to temperate marine forests and intertidal regions. The data were based on Wahl, et al. [71].

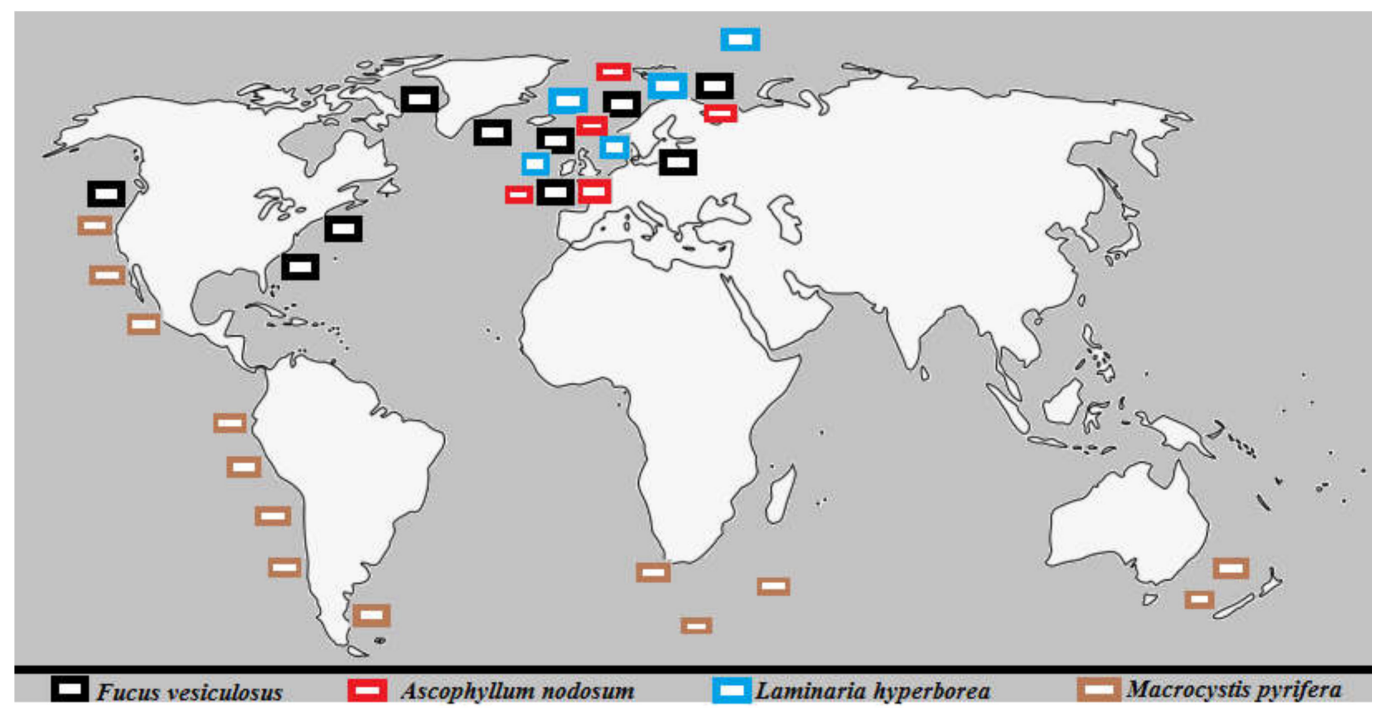

Figure 1. Global distribution of the major brown seaweeds' species. They dominate tropical to temperate marine forests and intertidal regions.

Furthermore, like terrestrial plant tissue culture (PTC), several biotechnological attempts were performed to cultivate and/or regenerate thallus from different species of brown seaweeds using seaweeds tissue culture [72]. They include micropropagation, callus induction and protoplast isolation [69,73-75]. They are very promising techniques as it may not only help to overcome the previously mentioned fucoidans production heterogeneity challenges [53] but also provide a sustainable supply [76]. However, compared to PTC, STC is still not well-enough established to be used for production of hydrocolloids and fucoidans [77] or cultivation in closed, well-controlled bioreactors, as in case of the red algae organism Agardhiella subulata [78].

\section{Downstream Processes}

Fucoidans are anionic polymers occurring in highly complicated matrices in cell walls and intercellular spaces along with other carbohydrate polymers (e.g., alginate, cellulose and laminarin), polyphenols and proteins [79]. Additionally, due to the sulfate ester groups, fucoidans are water soluble polysaccharide polymers [80] exhibiting high affinity to other cell wall components, especially polyphenols [81]. Therefore, various and complicated downstream processes are required to remove such extraneous substances before and after precipitation with ethanol or cationic surfactants to obtain high-purity fucoidans $[82,83]$. The processes always include pre-treatment, extraction and purification stages as shown in Figure 2. 


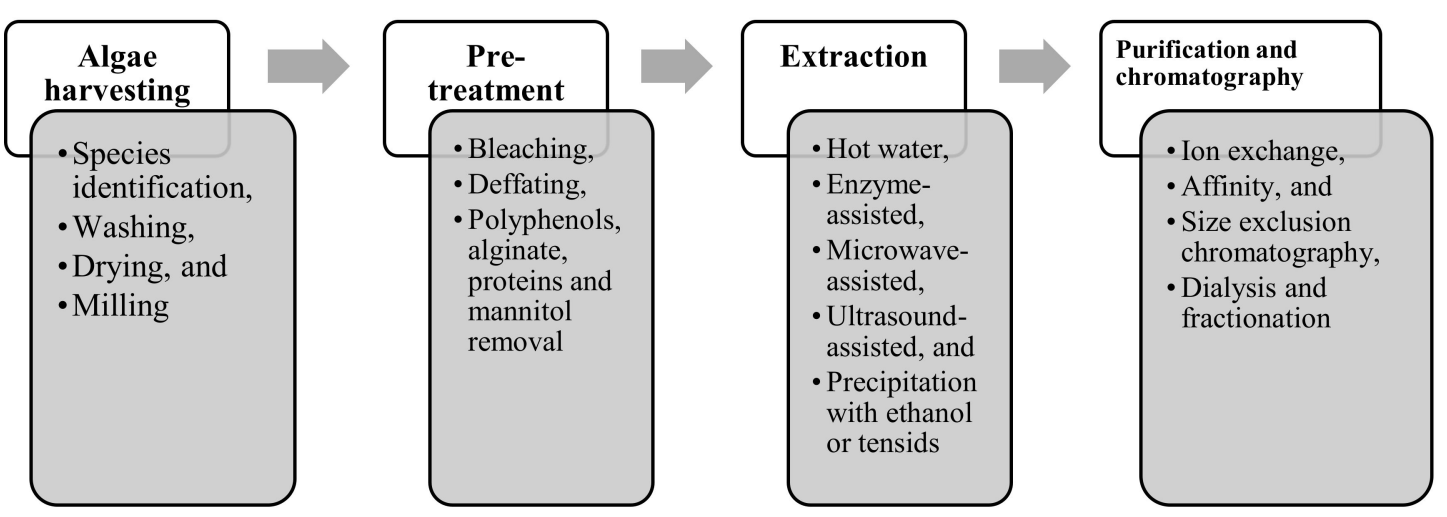

Figure 2. Required downstream processes including steps in each process for fucoidans production.

\subsection{Pre-Treatment}

After harvesting algal biomass from beaches, the biomass should be washed thoroughly with tap water to remove sands and epiphytes, then dried and milled to increase the area-to-mass ratio. Several pre-treatment steps are performed before the extraction step to release fucoidans from intercalating components, ease the following extraction process, improve the extraction yield, and decrease the possible interferences from co-extracted components in purification and biological investigations.

Previous experiments tried to remove pigments (e.g., chlorophyll, flavins and carotenoids) and lipids in specific bleaching and defatting steps with acetone, toluene, charcoal or $80 \%-85 \%(v / v)$ ethanol $[34,84,85]$. Since fucoidans are negatively charged molecules, they remained unaffected by incubation with organic solvents (e.g., acetone, toluene or hexane:isopropanol (3:2) mixture) during pre-treatment of the dried algal biomass. Such extracts were further treated to obtain carotenoids, represented by fucoxanthin in brown algae [86], lipids and fatty acid metabolites (especially essential polyunsaturated fatty acids (PUFA) and fucosterol), adding to nutraceutical applications of brown algae $[87,88]$. In contrast, activated carbon materials, such as charcoal, adsorb the target fucoidans molecules, adversely affecting the final production yield [79].

Other studies tried to exclude the tightly non-covalently bound polyphenolic compounds represented by phloroglucinol-type phlorotannins [89], which contribute to the light to dark brown color of the crude fucoidans extract (along with fucoxanthin) [41,81]. They reported comparatively high phlorotannins content, reaching approximately one fifth of the brown algae dry weight [25]. Phlorotannins perform major structural and physiological functions, like tannins found in plants, including defense against biotic and abiotic stresses [90,91]. Despite of the great pharmacological importance of phlorotannins [92,93], their presence in high-quality fucoidans is not acceptable because of the possibility of interference with the anti-oxidant [25,52,94] and anti-tumor activities of fucoidans [95]. Therefore, the natural phenolics content of fucoidans should be determined before the measurement of their biological activities [96]. Therefore, nearly all pre-extraction protocols for fucoidans involved strategies to remove such contaminants, e.g., incubation with EtOH: $\mathrm{H}_{2} \mathrm{O}: \mathrm{HCHO}(16: 3: 1)(v / v / v)$ at $\mathrm{pH}$ 2. Under such conditions, formaldehyde enhances the crosslinking and polymerization of such polyphenolic contaminants and the high volume of ethanol results in protein denaturation $[41,60,97,98]$. However, the toxicity of formaldehyde limits its utilization in pre-treatment protocols [51].

Furthermore, pre-treatment steps are performed to remove other carbohydrates such as alginate, the major hydrocolloids in brown algae [99]. This is commonly removed by formation of water-insoluble calcium complex either before [60] or during the extraction procedure using $1 \%-4 \%(w / v) \mathrm{CaCl}_{2}$ followed by a filtration or centrifugation step to remove the formed precipitate $[58,98,100,101]$. These previously mentioned procedures were optimized using successive incubation, centrifugation or filtration, washing and drying for the main extraction step of the dried, milled algal biomass, as described in Figure 3. The application of such an optimized protocol resulted in a dried, pre-treated powder 
representing $71 \%(w / w)$ of the starting material [98]. Despite these results, downstream processing of fucoidans, except with enzymatic modification, starts with a small scale (e.g., 5-10 g of the dried algal biomass) to optimize parameters like dried biomass to solvent ratio, temperatures, $\mathrm{pH}$, and incubation time, based on preliminary quality and yield of crude fucoidans measured by infra-red spectroscopy (IR), simple sugar tests and elemental analysis. After this, transfer to large scale production could be accomplished using larger biomass quantities (e.g., 500-1000 g).

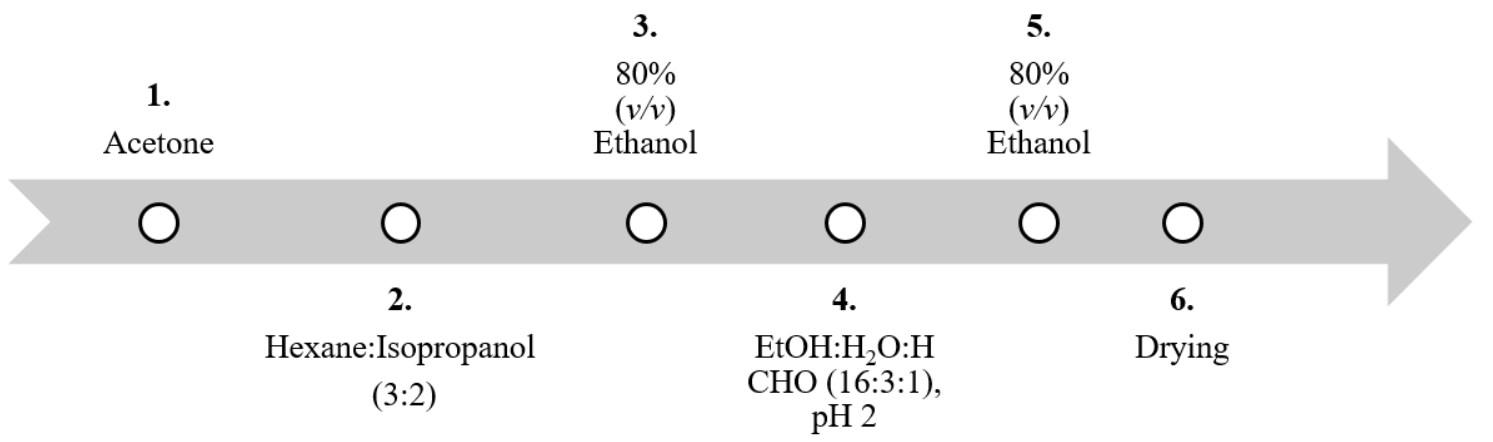

Figure 3. Overview of optimized pre-treatment steps of the dried algae biomass before fucoidans extraction. All steps were performed at $25^{\circ} \mathrm{C}$ overnight and the ratio between dried algal biomass to solvent was 1:10, except for the acetone step, which was 1:20 (modified after [98,102]).

Due to several complicated pre-treatment steps, general protocols always employ a single incubation step using the ternary mixture composed of $\mathrm{CH}_{3} \mathrm{OH}: \mathrm{CH}_{3} \mathrm{Cl}: \mathrm{H}_{2} \mathrm{O}(4: 2: 1)(\mathrm{v} / v / v)$ [103], binary mixture of $\mathrm{CH}_{2} \mathrm{Cl}_{2}: \mathrm{EtOH}(94.2: 5.8, v / v)$ [104], or aqueous ethanol (e.g., 95\% v/v) [105,106] to remove pigments [107], polyphenols [51,103] and lipids [108]. Nevertheless, pre-treatment steps may be insufficient for complete removal or prevention of some residual co-extraction.

Notably, all these procedures were carried out at room temperature in organic solvents and high volumes of ethanol, in which fucoidans are insoluble. Theoretically, the native structural backbone should not be affected. However, similar polymeric carbohydrates such as laminarin may still be present, contaminating the extract after these steps.

Recently, in order to decrease pollution of organic toxic solvents, compressional-puffing pre-treatment was applied for Sargassum hemiphyllum and S. glaucescens fucoidans. The pre-treatment method was based on mechanical pressure at higher temperatures that loosen the cell wall matrix before the step of extraction. Such methods succeeded in increasing the production yield, but they affected the molecular features of the fucoidans, including molecular weight $[109,110]$.

\subsection{Extraction}

As previously mentioned, fucoidans are principally anionic water-soluble macromolecules. Therefore, they can be extracted from the pre-treated biomass using a simple hot- or cold-water incubation. Afterwards, the extracted fucoidans can be precipitated by high volumes of solvents with a low dielectric constant (e.g., $>70 \%(v / v),>2.5$ volume ethanol [111,112], <2 volume acetone [113]) or cationic surfactants (e.g., hexadecyltrimethylammonium bromide (Cetavlon ${ }^{\circledR}$ ) 10\% (v/v)) [55] via an affinity complex formation at low temperatures $\left(4^{\circ} \mathrm{C}\right)$ to remove the undesired salts from the sulfated polysaccharides [52]. This specific precipitation reaction between fucoidans and Cetavlon ${ }^{\circledR}$ is applied in screening tests of microorganisms for putative fucoidanase activity [114].

Ale et al. published comprehensive articles discussing the history of extraction, including the different classical extraction methods of fucoidans, and reported that extraction procedures significantly affect the polymers monomeric composition, even for the same organism $[60,115]$. Beyond simple hot water extraction $[58,116]$, attempts were made to increase the selectivity and extraction yields, including extraction in acidic [117], alkaline [118], and buffered [41,119] aqueous solutions. However, a neutralization step is required, using $\mathrm{Na}_{2} \mathrm{CO}_{3}$ or $\left(\mathrm{NH}_{4}\right)_{2} \mathrm{CO}_{3}$, directly after extraction to guard against 
the non-specific acidic hydrolysis of the polymer [101,115]. Such drastic $\mathrm{pH}$ changes affect the chemical and physicochemical properties of fucoidans during the extraction step.

Currently, besides the previously discussed classical extraction methods based on thermal energy, extraction protocols based on vibrational energy have been developed. These protocols are based on microwave-assisted (MAE) $[120,121]$ or ultrasound-assisted (UAE) $[94,122]$ extraction steps to elicit cell wall degradation which improves the polymer release into aqueous solvent. These protocols were optimized either using an approach that modified one factor at a time or a multiple factorial design, setting the polymers production yield, monomeric composition and biological activities as the measured responses.

Recently, combined sulfated polysaccharides extraction protocols were optimized from different brown algae species using hydrothermal-assisted extraction (HAE) followed by sequential ultrasound and thermal technologies [123]. Similarly, subcritical water extraction was applied to increase the production yield of fucoidans from Nizamuddinia zanardinii [124]; such mild conditions may be advantageous to preserve the native chemical backbone and physicochemical characters of fucoidans.

Recently, as a trial to reduce such undesirable effects, enzyme-aided or assisted extraction (EAE) protocols are being developed using enzymes instead of harsh chemicals and high extraction temperatures during extraction. These include cellulase, papain, laminarinase, alginate lyase, and protease, which are present in products of Novozymes [79,125-128]. In addition, other cost-effective and time-saving techniques are reported, like those for terrestrial plant polysaccharides, such as extraction under vacuum to lower the boiling point of water and hence avoid possible heat-induced fucoidans degradation [129]. Alternatively, 0.5\% (w/v) ethylenediaminetetraacetic acid (EDTA) was applied at $70{ }^{\circ} \mathrm{C}$ for simultaneous extraction of Laminaria japonica fucoidans and removal of pigments [130].

\subsection{Separation Physical Methods}

Filtration, dialysis and centrifugation, either for the algal biomass or precipitates, are also among the downstream processes after pre-treatment and extraction steps [131-133]. Cross-flow filtration and dialysis against water are usually performed using different molecular weight cut-off (MWCO) membranes for isolation of fucoidans from smaller compounds depending on the high molecular weight of fucoidans [134] and also for fractionation purposes, where low molecular weight fucoidans (LMWF) can be separated from high molecular weight analogues (HMWF) [49].

In addition, filtration, concentration, and fractionation are simultaneously performed using centrifugal concentrators (Vivaspin ${ }^{\circledR}$ ) equipped with membranes with certain MWCO, like in protein purification. However, in some cases, especially in the presence of bulk masses or high concentrations of salts and small contaminants, the use of centrifugal concentrators becomes practically and economically unsuitable for fucoidans purification. In such cases, bulky contaminants result in membrane clogging leading to its deterioration and increasing the production cost.

\subsection{Purification}

Despite the previously mentioned purification steps, residuals of co-extracted contaminants are still present, and resulting fucoidans are still crude-type. [27]. Therefore, further selective purification steps are needed to obtain a high-quality product for reproducible and accurate biological investigations. Some researches adopted simple, non-chromatographic steps, such as bleaching of the crude fucoidans $\left(\mathrm{NaClO}_{2}\right.$ in dilute $\left.\mathrm{HCl}\right)$ followed by precipitation with cetyltrimethylammonium bromide [135] or by cold overnight incubation in aqueous buffered solution of calcium acetate $(20 \mathrm{mM}, \mathrm{pH}=6.5$ -7.5) followed by dialysis [136]. In addition, membrane filtration was reported to produce fucoidans fractions of different molecular weight [137].

However, other chromatographic purification techniques were discussed in our previous publications $[41,53,98,102]$. Almost all the chromatographic techniques are based on the permanent negatively charged sulfate ester groups distributed on the polymer backbone which allow selective 
fucoidans capture. However, carboxylated (e.g., alginate) and phosphorylated (e.g., nucleic acids) compounds might interfere $[138,139]$. Therefore, the $\mathrm{pH}$ value of the applied solvents is critical during chromatographic purification. One option for this uses anionic exchange resins (e.g., diethylaminoethyl cellulose or DEAE-cellulose), which was performed at $\mathrm{pH} 7.2$ using $0.1 \mathrm{M}$ sodium phosphate buffer [140]. An alternative is cationic dyes (e.g., toluidine blue- or perylene diimide derivative), modified resins or chitosan functioning in buffered solutions $[27,102]$. Both anion exchange and dye affinity chromatography involve the use of highly concentrated $\mathrm{NaCl}$ elution solvents. As a result, a subsequent purification step using chromatographic gel permeation [141] or dialysis [140] is required to remove salts, increasing the production costs. Other methods based on the use of biological macromolecules, such as lectins and anti-thrombin III, were also reported [53].

Novel innovative purification techniques were recently developed, such as selective solid phase extraction for purifying fucoidans and other complex seaweeds polymers by molecularly imprinted polymers (MIP) [142,143] or MIP modified by deep eutectic solvents [142,143]. Abdella et al., developed a green and time-saving purification protocol using genipin cross-linked toluidine blue immobilized-chitosan beads employing fucoidans affinity to cationic thiazine dyes [102].

\section{Recent Uncommon Applications}

In addition to the classical therapeutic applications of fucoidans, including anti-coagulant [41,144], anti-viral $[145,146]$, anti-inflammatory $[46,147]$ and selective cytotoxic and anti-tumor uses $[39,50]$, uncommon bioactivities, including cosmeceutical, pharmaceutical, diagnostic, and synergistic therapeutic applications were recently reported [32]. Recent fucoidans uses included therapeutic treatment of major blindness diseases [148]. It has also been used as a drug carrier, especially for anti-cancer treatments and anti-biotics. Additionally, fucoidans have been shown to improve drug bioavailability and efficacy in pharmaceutical formulations, including in nanoparticles, liposomes, microparticles, and semisolid formulations $[28,149,150]$. Table 1 summarizes some of the recent and uncommon fucoidans applications based on in-vitro or in-vivo studies, in addition to biogenic resources and physicochemical features. 
Table 1. Some selected recent therapeutic, diagnostic and pharmaceutical applications of fucoidans including the biogenic sources.

\begin{tabular}{|c|c|c|c|c|c|}
\hline Application & $\begin{array}{l}\text { Biogenic } \\
\text { Source }\end{array}$ & $\begin{array}{c}\text { Quality } \\
\text { Grade/Purification } \\
\text { Method }\end{array}$ & Structural Features & Involved Mechanism & Ref. \\
\hline \multicolumn{6}{|c|}{ Therapeutic } \\
\hline $\begin{array}{l}\text { Anti-viral } \\
\text { (IAV) }\end{array}$ & $\begin{array}{l}\text { Kjellmaniella } \\
\text { crassifolia } \\
\text { (Laminariales) }\end{array}$ & * & & $\begin{array}{c}\text { Inhibition of the viral } \\
\text { neuraminidase (NA) } \\
\text { Interference with the cellular } \\
\text { EGFR pathway }\end{array}$ & [43] \\
\hline $\begin{array}{l}\text { Anti-metabolic } \\
\text { syndrome }\end{array}$ & \multirow[t]{3}{*}{$\begin{array}{l}\text { Fucus vesiculosus } \\
\text { (Fucales) }\end{array}$} & $\begin{array}{l}\text { Dialysis of crude } \\
\text { alginate-free fucoidans }\end{array}$ & $\begin{array}{c}\text { Alternating } \\
\alpha(1 \rightarrow 3) / \alpha(1 \rightarrow 4) \text { linked } \\
\text { fucose, } \\
\mathrm{Mw}>7.0 \times 10^{3} \mathrm{~g} / \mathrm{mol}\end{array}$ & $\begin{array}{l}\text { Regulation of jnk, akt, } \\
\text { and ampk signaling } \\
\text { Alleviation of insulin } \\
\text { resistance Regulation of } \\
\text { lipid metabolism }\end{array}$ & [151] \\
\hline \multirow[t]{2}{*}{ Anti-leishmaniasis } & & $\begin{array}{l}\text { Commercial product } \\
\text { purchased from } \\
\text { Sigma-Aldrich }^{\circledR}\end{array}$ & $\begin{array}{l}\text { Polymer of } \alpha-(1 \rightarrow 3) \\
\quad \text { linked fucose }\end{array}$ & $\begin{array}{c}\text { Activation of the } \\
\text { mitogen-activated protein } \\
\text { kinase (MAPK)/NF-KB } \\
\text { pathway against Leishmania } \\
\text { donovani-infected } \\
\text { macrophages }\end{array}$ & [152] \\
\hline & & & & $\begin{array}{l}\text { Enhancement of dendritic } \\
\text { cells maturation, production } \\
\text { of } \\
\text { pro-inflammatory cytokines, } \\
\text { and down-regulation of } \\
\text { anti-inflammatory cytokines }\end{array}$ & {$[153,154]$} \\
\hline Immunostimulant & $\begin{array}{l}\text { Nizamuddinia } \\
\text { zanardinii } \\
\text { (Fucales) }\end{array}$ & $\begin{array}{l}\text { A fraction of DEAE } \\
\text { Sepharose Fast Flow } \\
\text { column }\end{array}$ & $\begin{array}{c}\text { Highly branched } \\
\text { polymer } \\
\text { Mw: } 953.6 \times 10^{3} \mathrm{~g} / \mathrm{mol}\end{array}$ & $\begin{array}{c}\text { Stimulation of RAW264.7 } \\
\text { murine macrophage and NK } \\
\text { cells }\end{array}$ & [155] \\
\hline Anti-metastasis & \multirow[t]{3}{*}{$\begin{array}{c}\text { Undaria } \\
\text { pinnatifida } \\
\text { (Laminariales) }\end{array}$} & $\begin{array}{l}\text { DEAE-cellulose, } \\
\text { and Sephadex G-100 } \\
\text { column } \\
\text { chromatography } \\
\text { (purity }>90 \%)\end{array}$ & $\begin{array}{c}\text { Mw: of } 10.4356 \times 10^{4} \\
\mathrm{~g} / \mathrm{mol}\end{array}$ & $\begin{array}{l}\text { - Suppression of Hca-F cell } \\
\text { growth, adhesion, invasion, } \\
\text { and metastasis capabilities, } \\
\text { - Inactivation of the NF- } \mathrm{kB} \\
\text { pathway }\end{array}$ & [156] \\
\hline $\begin{array}{l}\text { Gastrointestinal } \\
\text { tract protective }\end{array}$ & & $\begin{array}{c}\text { Purity } \geq 95 \% \\
\text { (Commercial product } \\
\text { purchased from } \\
\text { Sigma-Aldrich }{ }^{\circledR} \text { ) }\end{array}$ & & $\begin{array}{c}\text { Protection against } \\
\mathrm{H}_{2} \mathrm{O}_{2} \text {-induced damage via } \\
\text { activation of the NRF2 } \\
\text { signaling pathway }\end{array}$ & [157] \\
\hline Anti-malaria & & $\begin{array}{l}\text { - Partial purification by } \\
\text { cetylpyridinum } \\
\text { chloride } \\
\text { Fractionation by } \\
\text { DEAE-Sephadex A-25 } \\
\text { column }\end{array}$ & $\begin{array}{l}\text { Sugar monomers, } \\
\text { and uronic acid, } \\
\text { M.wt: approx. } 15 \times \\
10^{3} \mathrm{~g} / \mathrm{mol}\end{array}$ & $\begin{array}{c}\text { In-vitro and in-vivo } \\
\text { inhibition of erythrocytes } \\
\text { invasion by P. falciparum } \\
\text { merozoites }\end{array}$ & [158] \\
\hline $\begin{array}{c}\text { Renal } \\
\text { protective }\end{array}$ & \multirow[t]{2}{*}{$\begin{array}{c}\text { Laminaria } \\
\text { japonica } \\
\text { (Laminariales) }\end{array}$} & & $\begin{array}{c}\text { LMWF } \\
\left(\mathrm{Mw}: 7 \times 10^{3} \mathrm{~g} / \mathrm{mol}\right)\end{array}$ & $\begin{array}{l}\text { Inhibition of overexpression } \\
\text { of pro-inflammatory and } \\
\text { pro-fibrotic factors, oxidative } \\
\text { stress and apoptosis }\end{array}$ & {$[159,160]$} \\
\hline $\begin{array}{l}\text { Cardio-, } \\
\text { hepatic- and } \\
\text { renal protective }\end{array}$ & & $\begin{array}{l}\text { Commercial product } \\
\text { purchased from } \\
\text { Absunutrix } \\
\text { Lyfetrition }^{\circledR}\end{array}$ & & $\begin{array}{l}\text { Reduction of oxidative } \\
\text { stress, pro-inflammatory } \\
\text { effects and injuries to the } \\
\text { cardiac, hepatic, and renal } \\
\text { tissues }\end{array}$ & [161] \\
\hline $\begin{array}{l}\text { Inhibition of } \\
\text { tumor } \\
\text { angiogenesis }\end{array}$ & $\begin{array}{l}\text { Sargassum } \\
\text { hemiphyllum } \\
\text { (Fucales) }\end{array}$ & $\begin{array}{l}\text { Hydrolyzed crude } \\
\text { extract }\end{array}$ & $\begin{array}{l}\text { LMWF; } \\
760 \mathrm{~g} / \mathrm{mol}\end{array}$ & $\begin{array}{c}\text { Suppression of } \\
\text { HIF-1/VEGF-regulated } \\
\text { signaling pathway }\end{array}$ & [162] \\
\hline Pro-angiogenic & $\begin{array}{l}\text { Ascophyllum } \\
\text { nodosum } \\
\text { (Fucales) }\end{array}$ & $\begin{array}{l}\text { Fractionated with } \\
\text { dialysis commercial } \\
\text { crude fucoidan } \\
\text { (ASPHY) }\end{array}$ & $\begin{array}{c}\text { LMWF } \\
\left(<4.9 \times 10^{3} \mathrm{~g} / \mathrm{mol}\right)\end{array}$ & $\begin{array}{l}\text { Increase of the vascular } \\
\text { network formation } \\
\text { regulated via Erk1/2 and } \\
\text { PI3K/AKT cell signaling } \\
\text { pathways }\end{array}$ & [163] \\
\hline $\begin{array}{l}\text { Alleviation of } \\
\text { diabetic } \\
\text { complications }\end{array}$ & $\begin{array}{l}\text { S. Fusiforme } \\
\text { (Fucales) }\end{array}$ & Crude extract & $\begin{array}{l}\text { Mw: } 205.89 \times 10^{3} \mathrm{~g} / \mathrm{mol} \text {, } \\
\text { high sulfate content }\end{array}$ & $\begin{array}{l}\text { - Suppression of oxidative } \\
\text { stress } \\
\text { - Alteration of the gut } \\
\text { microbiota } \\
\text { - Attenuation of the } \\
\text { pathological changes in } \\
\text { heart and liver }\end{array}$ & [164] \\
\hline
\end{tabular}


Table 1. Cont.

\begin{tabular}{|c|c|c|c|c|c|}
\hline Application & $\begin{array}{l}\text { Biogenic } \\
\text { Source }\end{array}$ & $\begin{array}{c}\text { Quality } \\
\text { Grade/Purification } \\
\text { Method }\end{array}$ & Structural Features & Involved Mechanism & Ref. \\
\hline \multicolumn{6}{|c|}{ Diagnostic } \\
\hline \multirow[t]{2}{*}{$\begin{array}{l}\text { Imaging of } \\
\text { cardiovascular } \\
\text { diseases }\end{array}$} & $\begin{array}{l}\text { Ascophyllum } \\
\text { nodosum } \\
\text { (Fucales) }\end{array}$ & $\begin{array}{c}\text { An oxidative-reductive } \\
\text { degraded crude extract } \\
\text { (purchased from } \\
\text { Algues and Mer, } \\
\text { Ascophyscient }{ }^{\circledR} \text { ) }\end{array}$ & $\begin{array}{l}\text { GMP-grade LMWF } \\
\left(7.1 \times 10^{3} \mathrm{~g} / \mathrm{mol}\right)\end{array}$ & $\begin{array}{l}\text { Synthesis of } \\
\text { technetium-99m-fucoidan } \\
\text { radiotracer for detection of } \\
\text { P-selectin }\end{array}$ & [56] \\
\hline & & $\begin{array}{l}\text { Commercial product } \\
\text { from Algues and Mer }\end{array}$ & & $\begin{array}{c}\text { Synthesis of } \\
\text { polycyanoacrylate-fucoidan } \\
\text { microcapsules (Fuco-MCs) } \\
\text { for detection of P-selectin }\end{array}$ & [165] \\
\hline \multicolumn{6}{|c|}{ Cosmeceutical } \\
\hline Anti-Photoaging & $\begin{array}{l}\text { Ecklonia cava } \\
\text { (Laminariales) }\end{array}$ & $\begin{array}{c}\text { Enzymatic } \\
\text { degradation of } \\
\text { a commercial HMWF }\end{array}$ & $\begin{array}{l}\text { LMWF (Mw: } 8 \times 10^{3} \\
\text { g/mol) }\end{array}$ & $\begin{array}{c}\text { Anti-oxidant, anti-apoptotic, } \\
\text { and MMP-9-inhibiting } \\
\text { effects }\end{array}$ & [166] \\
\hline $\begin{array}{c}\text { Skin } \\
\text { brightening } \\
\text { and age spot } \\
\text { reduction }\end{array}$ & $\begin{array}{l}\text { F. vesiculosus } \\
\text { (Fucales) }\end{array}$ & \multirow[t]{2}{*}{$\begin{array}{l}\text { Crude extracts } \\
\text { purchased from } \\
\text { Marinova }{ }^{\circledR} \text { Pty Ltd. }\end{array}$} & $\begin{array}{l}58.6 \% \text { fucoidans, } \\
33.7 \% \text { polyphenol }\end{array}$ & \multirow[t]{2}{*}{$\begin{array}{l}\text { Increase of Sirtuin } 1 \text { (SIRT1) } \\
\text { expression in vitro }\end{array}$} & \multirow[t]{2}{*}[167,168]{} \\
\hline $\begin{array}{l}\text { Skin immunity, } \\
\text { soothing and } \\
\text { protection }\end{array}$ & $\begin{array}{l}\text { U. pinnatifida } \\
\text { (Laminariales) }\end{array}$ & & $\begin{array}{l}89.6 \% \text { fucoidans, } \\
<2 \% \text { polyphenol }\end{array}$ & & \\
\hline $\begin{array}{l}\text { Reconstruction } \\
\text { of skin }\end{array}$ & $\begin{array}{l}\text { F. vesiculosus } \\
\text { (Fucales) }\end{array}$ & $\begin{array}{l}\text { Commercial product } \\
\text { from Sigma-Aldrich }{ }^{\circledR} \\
\text { (not determined the } \\
\text { degree of purity) }\end{array}$ & & $\begin{array}{l}\text { Increase of proliferating cell } \\
\text { nuclear antigen (PCNA) p63 } \\
\text { and } \alpha 6 \text {-integrin expression }\end{array}$ & [169] \\
\hline \multicolumn{6}{|c|}{ Pharmaceutical technology } \\
\hline \multirow[t]{3}{*}{$\begin{array}{l}\text { As vehicle for } \\
\text { drug delivery }\end{array}$} & \multirow[t]{4}{*}{$\begin{array}{l}\text { F. vesiculosus } \\
\text { (Fucales) }\end{array}$} & \multirow[t]{4}{*}{$\begin{array}{l}\text { Commercial product } \\
\text { purchased from } \\
\text { Sigma-Aldrich }^{\circledR}\end{array}$} & Mw: $57.26 \times 10^{3} \mathrm{~g} / \mathrm{mol}$ & $\begin{array}{l}\text { - Chitosan-fucoidans-based } \\
\text { nanoparticles for delivery of } \\
\text { anti-cancers (e.g., } \\
\text { curcumin-loaded NPs) } \\
\text { - Nanoencapsulation of poly } \\
\text { L-lysine }\end{array}$ & {$[170,171]$} \\
\hline & & & & $\begin{array}{l}\text { Piperlongumine (PL)-loaded } \\
\text { chitosan-fucoidan } \\
\text { nanoparticles (PL-CS-F NPs) }\end{array}$ & [172] \\
\hline & & & & $\begin{array}{c}\text { Synthesis of } \\
\text { fucoidan/trimethylchitosan } \\
\text { nanoparticles } \\
\text { (FUC-TMC-NPs) as adjuvant } \\
\text { in anthrax vaccine adsorbed }\end{array}$ & [173] \\
\hline $\begin{array}{l}\text { Green synthesis } \\
\text { of silver } \\
\text { nanoparticles }\end{array}$ & & & & $\begin{array}{c}\text { Synthesis of } \\
\text { chitosan-fucoidan } \\
\text { complex-coated AgNPs }\end{array}$ & [174] \\
\hline
\end{tabular}

*: Not specified.

\section{Enzymatic Modification of Native Fucoidans}

Owing to their high molecular weight, therapeutic applications of native fucoidans face many challenges including structure elucidation, solubility, manufacturing, and handling [63,116], in addition to safety as a food supplement [175]. Structure elucidation and quantitation of native fucoidans is highly complicated and requires advanced or hyphenated spectroscopic techniques such asHPLC-MS/MS as it applied in Sea Cucumbers fucoidans $[176,177]$. Also, these techniques must be applied after a step of enzymatic or acid hydrolysis to transform the fucoidans polymers to oligomers. According to their molecular weight, fucoidans are classified into three classes: LMWF ( $<10 \mathrm{kDa})$, medium molecular weight fucoidan (MMWF) (10-10000 kDa), and HMWF (>10000 kDa) [31]. LMWF demonstrated better bioavailability and bioactivities than HMWF $[178,179]$. As a consequence, several articles reported physical, chemical and enzymatic modification of the native HMWF to get LMWF of higher biological activity [62]. Specifically, enzymatic modification of macroalgal polysaccharides, including fucoidans by either fucoidanases or sulfatases, is characterized by regioselectivity and 
stereospecificity. This new trend is considered crucial and highly promising for current and future applications of polysaccharides [180].

Nevertheless, our publications in 2009 particularly reviewed the specific enzymatic degradation of fucoidans induced by fucoidanases (EC 3.2.1.44) and $\alpha{ }_{-}-$-fucosidases (EC 3.2.1.51), mainly those isolated from marine bacteria [35]. Cumashi, et al. studied the chemical structures of different fucoidans isolated from a number of brown algal species [181]. Their proposed models, which were highly appreciated and recommended by many researchers [60], showed the backbone of fucoidans to be mainly an alternating $\alpha-(1-4)$ and $\alpha-(1-3)$ linked $_{\mathrm{L}}$-fucopyranoside. Regarding the sulfation pattern, C-2 is usually substituted with sulfate ester groups in addition to alternating C-3 or C-4 in L-fucopyranose residue, according to the glycosidic linkages. In addition, branched chain polymers were also found as in F. serratus. Other minor sugar units (e.g., mannose, galactose, glucose and xylose) occur as well in fucoidans structure; however, their distribution pattern and positions are still unknown $[60,181]$. Now, the mechanism of enzymatic degradation can be described in relation to fucoidans chemical structures.

Despite the increasing number of publications investigating fucoidanase activity of different marine species cell extracts, few of these enzymes have been isolated and characterized. Moreover, genome sequences encoding few fucoidanases have been published, including Ffa2 and FFA1 from Formosa algae KMM $3553^{\mathrm{T}}$ [182,183], FcnA from Mariniflexili fucanivorans SW5T [184]. Therefore, specificity of fucoidanases, type of cleaved glycoside bond, structure-activity relationship studies and enzyme stability are still poorly described. It was only observed that identified microbial fucoidanses act only on fucoidans isolated from their respective symbionts [185]. In fact, fucoidanases have not actually been fully utilized yet as a powerful tool either for the structural studies of fucoidans or production of defined and well-characterized bioactive fragments of extracted fucoidans, as shown in Table 2.

Similarly, recent advances in bioinformatics and genome sequencing of microbial species have resulted in a continual increase of novel genome sequences. These genomes demonstrated various potential genes encoding for enzymes with biopolymer-degrading capabilities, such as Shewanella violacea DSS12 (NC_014012.1), Formosa algae KMM 3553 (NZ_LMAK01000014.1) [182], Formosa haliotis MA1 (NZ_BDEL01000001.1) [198], Wenyingzhuangia fucanilytica CZ1127 (NZ_CP014224.1) [199] and Pseudoalteromonas sp. strain A601 (MXQF01000000) [200]. Moreover, production of stabilized fucoidanases has been achieved by targeted truncation of the C-terminal of FcnA2, Fda1 and Fda2. This recently developed method may help with enzymatic production of defined degrees of polymerization and more bioactive products from native fucoidan substrates [201]. 
Table 2. Source of fucoidans as a substrate and mode of action of some fucoidanases.

\begin{tabular}{|c|c|c|c|}
\hline $\begin{array}{l}\text { Biogenic Source of } \\
\text { Fucoidans }\end{array}$ & Fucoidanase Source & Mode of Action & Ref. \\
\hline \multirow[t]{2}{*}{ F. evanescens } & Formosa algae KMM 3553 & $\begin{array}{l}\text { Endo } \\
\alpha-1 \rightarrow 4\end{array}$ & {$[61,182]$} \\
\hline & $\begin{array}{c}\text { Pseudoalteromonas citrea } \\
\text { strains KMM 3296, KMM 3297, } \\
\text { KMM } 3298\end{array}$ & $\underset{\alpha-1 \rightarrow 3}{\text { Endo }}$ & [186] \\
\hline F. vesiculosus & Dendryphiella. arenaria TM94 & $\begin{array}{l}\text { Endo } \\
\text { n.d. * }\end{array}$ & [187] \\
\hline Kjellmaniella crassifolia & Fucobacter marina SA-0082 & $\begin{array}{c}\text { Endo } \\
\beta-1 \rightarrow 4\end{array}$ & [188] \\
\hline \multirow[t]{2}{*}{ Cladosiphon okamuranus } & $\begin{array}{l}\text { Fucophilus fucoidanolyticus } \\
\text { SI-1234 }\end{array}$ & $\begin{array}{l}\text { Endo } \\
\alpha-1 \rightarrow 3\end{array}$ & [189] \\
\hline & Flavobacterium sp. F-31 & $\begin{array}{c}\text { Endo } \\
\text { n.d. }\end{array}$ & [190] \\
\hline F. distichus & Littorina kurila & $\begin{array}{c}\text { Endo } \\
\alpha-1 \rightarrow 3\end{array}$ & [191] \\
\hline Pelvetia canaliculata & Mariniflexile fucanivorans SW5 ${ }^{\mathrm{T}}$ & $\begin{array}{c}\text { Endo } \\
\alpha-1 \rightarrow 4\end{array}$ & [184] \\
\hline Undara pinnatifida & Sphingomonas paucimobilis PF-1 & $\begin{array}{l}\text { Endo } \\
\text { n.d. }\end{array}$ & {$[192,193]$} \\
\hline Saccharina cichorioides & $\begin{array}{c}\text { Pseudoalteromonas citrea } \\
\text { strains KMM 3296, KMM 3297, } \\
\text { KMM } 3298\end{array}$ & $\begin{array}{c}\text { Endo } \\
\alpha-1 \rightarrow 3\end{array}$ & [186] \\
\hline Nemacystus decipiens & Mizuhopecten yessoensis & $\begin{array}{l}\text { Endo } \\
\text { n.d. }\end{array}$ & [194] \\
\hline Ascophyllum nodosum & Pecten maximus & $\begin{array}{l}\text { Exo } \\
\text { n.d. }\end{array}$ & {$[195,196]$} \\
\hline $\begin{array}{l}\text { Thelenota ananas (Wild } \\
\text { sea cucumber) }\end{array}$ & Wenyingzhuangia Fucanilytica & $\begin{array}{l}\text { Endo } \\
\text { n.d. }\end{array}$ & [197] \\
\hline
\end{tabular}

\section{Conclusion and Future Prospective}

As multifunctional molecules, fucoidans have received special interest based on their proven efficacy in different fields. The current article reviewed many aspects related to fucoidans' production, mainly from brown algae. Biogenic source and downstream processes were shown as major factors determining their application, which is affected by molecular weight and quality grade of fucoidans. Therefore, the alteration of fucoidans' native structure was recommended, especially as performed by fucoidanases. Their production in nanoform or in combination with other polymers can improve or modify their potential uses, allowing their expanded potential as therapeutic agents, e.g., in anti-cancer applications [202].

Production of high-quality purified fucoidans is urgently required to clarify the relationships between chemical structure and the various bioactivities attributed to fucoidans, eliminating any interference from contaminants. However, it was observed in some cases that crude extracts and presence of co-extracted contaminants, especially polyphenolic phlorotannins, have advantageous cosmeceutical effects due to their powerful anti-oxidant activity [203,204].

Novel techniques, either in cultivation or downstream processes, have been established, increasing the global production yields and reducing ecological and economic problems. A new advance toward achieving such goals was established by optimization of water extraction via measurement of kinetic parameters [205]. In addition to this, it is expected that most future trends in marine biotechnology research will focus on the cell wall and extracellular matrix components of 
brown algae, including fucoidans' biosynthetic genes and production regulators [23,53,63,206-208]. Such trials may enable the scientific community to produce more bioactive molecules of fucoidans with defined characteristics, including degree of polymerization, sulfate content and pattern, in reproducible manners.

Author Contributions: R.U. planned the manuscript's topics and is the corresponding author, while A.Z. collected the data and wrote the article. All authors have read and agreed to the published version of the manuscript.

Acknowledgments: The research is funded by the „Deutsche Forschungsgemeinschaft (DFG, German Research Foundation)-Project-ID 172116086-SFB 926“. The authors would like also to thank Mrs. Aya Abdella and Ms. Gabrielle Phillips for helpful comments and English editing of the manuscript.

Conflicts of Interest: The authors declare no conflicts of interests.

\section{References}

1. Munoz-Bonilla, A.; Echeverria, C.; Sonseca, A.; Arrieta, M.P.; Fernandez-Garcia, M. Bio-based polymers with antimicrobial properties towards sustainable development. Materials 2019, 12, 641. [CrossRef] [PubMed]

2. Amos, R.A.; Mohnen, D. Critical review of plant cell wall matrix polysaccharide glycosyltransferase activities verified by heterologous protein expression. Front. Plant Sci. 2019, 10, 915. [CrossRef]

3. Lampugnani, E.R.; Flores-Sandoval, E.; Tan, Q.W.; Mutwil, M.; Bowman, J.L.; Persson, S. Cellulose synthesis-central components and their evolutionary relationships. Trends Plant Sci. 2019, 24, 402-412. [CrossRef] [PubMed]

4. Helle, S.; Bray, F.; Verbeke, J.; Devassine, S.; Courseaux, A.; Facon, M.; Tokarski, C.; Rolando, C.; Szydlowski, N. Proteome analysis of potato starch reveals the presence of new starch metabolic proteins as well as multiple protease inhibitors. Front. Plant Sci. 2018, 9, 746. [CrossRef] [PubMed]

5. Ball, S.; Colleoni, C.; Cenci, U.; Raj, J.N.; Tirtiaux, C. The evolution of glycogen and starch metabolism in eukaryotes gives molecular clues to understand the establishment of plastid endosymbiosis. J. Exp. Bot. 2011, 62, 1775-1801. [CrossRef] [PubMed]

6. Edmond Ghanem, M.; Han, R.-M.; Classen, B.; Quetin-Leclerq, J.; Mahy, G.; Ruan, C.-J.; Qin, P.; Pérez-Alfocea, F.; Lutts, S. Mucilage and Polysaccharides in the Halophyte plant species Kosteletzkya virginica: Localization and composition in relation to salt stress. J. Plant Physiol. 2010, 167, 382-392. [CrossRef]

7. Shukla, P.S.; Mantin, E.G.; Adil, M.; Bajpai, S.; Critchley, A.T.; Prithiviraj, B. Ascophyllum nodosum-based biostimulants: sustainable applications in agriculture for the stimulation of plant growth, stress tolerance, and disease management. Front. Plant Sci. 2019, 10, 655. [CrossRef]

8. Hamed, I.; Özogul, F.; Özogul, Y.; Regenstein, J.M. Marine bioactive compounds and their health benefits: A review. Compr. Rev. Food Sci. Food Saf. 2015, 14, 446-465. [CrossRef]

9. Lee, Y.-E.; Kim, H.; Seo, C.; Park, T.; Lee, K.B.; Yoo, S.Y.; Hong, S.C.; Kim, J.T.; Lee, J. Marine polysaccharides: therapeutic efficacy and biomedical applications. Arch. Pharmacal Res. 2017, 40, 1006-1020. [CrossRef]

10. Ruocco, N.; Costantini, S.; Guariniello, S.; Costantini, M. Polysaccharides from the marine environment with pharmacological, cosmeceutical and nutraceutical potential. Molecules 2016, 21, 551. [CrossRef]

11. Laurienzo, P. Marine Polysaccharides in Pharmaceutical Applications: An Overview. Mar. Drugs 2010, 8 , 2435-2465. [CrossRef] [PubMed]

12. Meenakshi, S.; Umayaparvathi, S.; Saravanan, R.; Manivasagam, T.; Balasubramanian, T. Hepatoprotective effect of fucoidan isolated from the seaweed turbinaria decurrens in ethanol intoxicated rats. Int. J. Biol. Macromol. 2014, 67, 367-372. [CrossRef] [PubMed]

13. Cunha, L.; Grenha, A. Sulfated seaweed polysaccharides as multifunctional materials in drug delivery applications. Mar. Drugs 2016, 14, 42. [CrossRef] [PubMed]

14. Hudak, J.E.; Bertozzi, C.R. Glycotherapy: New advances inspire a reemergence of glycans in medicine. Chem. Biol. 2014, 21, 16-37. [CrossRef] [PubMed]

15. Novotny, M.V.; Alley, W.R., Jr. Recent trends in analytical and structural glycobiology. Curr. Opin. Chem. Biol. 2013, 17, 832-840. [CrossRef] [PubMed]

16. Caldwell, G.S.; Pagett, H.E. Marine glycobiology: Current status and future perspectives. Mar. Biotechnol. 2010, 12, 241-252. [CrossRef] 
17. Pomin, V.H. Marine medicinal Gglycomics. Front. Cell. Infect. Microbiol. 2014, 4, 5. [CrossRef]

18. De Jesus Raposo, M.F.; de Morais, A.M.B.; de Morais, R.M.S.C. Marine polysaccharides from algae with potential biomedical applications. Mar. Drugs 2015, 13, 2967-3028. [CrossRef]

19. Gobet, A.; Barbeyron, T.; Matard-Mann, M.; Magdelenat, G.; Vallenet, D.; Duchaud, E.; Michel, G. Evolutionary evidence of algal polysaccharide degradation acquisition by Pseudoalteromonas carrageenovora $9^{\mathrm{T}}$ to adapt to macroalgal niches. Front. Microbiol. 2018, 9, 2740. [CrossRef]

20. Maneein, S.; Milledge, J.J.; Nielsen, B.V.; Harvey, P.J. A Review of seaweed pre-treatment methods for enhanced biofuel production by anaerobic digestion or fermentation. Fermentation 2018, 4, 100. [CrossRef]

21. Afonso, N.C.; Catarino, M.D.; Silva, A.M.S.; Cardoso, S.M. Brown macroalgae as valuable food ingredients. Antioxidants 2019, 8, 365. [CrossRef]

22. Catarino, M.D.; Silva, A.M.S.; Cardoso, S.M. Phycochemical constituents and biological activities of Fucus spp. Mar. Drugs 2018, 16, 249. [CrossRef]

23. Deniaud-Bouët, E.; Hardouin, K.; Potin, P.; Kloareg, B.; Hervé, C. A review about brown algal cell walls and fucose-containing sulfated polysaccharides: Cell wall context, biomedical properties and key research challenges. Carbohydr. Polym. 2017, 175, 395-408. [CrossRef] [PubMed]

24. Deniaud-Bouët, E.; Kervarec, N.; Michel, G.; Tonon, T.; Kloareg, B.; Hervé, C. Chemical and enzymatic fractionation of cell walls from Fucales: Insights into the structure of the extracellular matrix of brown algae. Ann. Bot. 2014, 114, 1203-1216. [CrossRef] [PubMed]

25. Generalić Mekinić, I.; Skroza, D.; Šimat, V.; Hamed, I.; Čagalj, M.; Popović Perković, Z. Phenolic content of brown algae (Pheophyceae) species: Extraction, identification, and quantification. Biomolecules 2019, 9, 244. [CrossRef] [PubMed]

26. Chang, Y.; Xue, C.; Tang, Q.; Li, D.; Wu, X.; Wang, J. Isolation and characterization of a sea cucumber fucoidan-utilizing marine bacterium. Lett. Appl. Microbiol. 2010, 50, 301-307. [CrossRef]

27. Zayed, A.; Dienemann, C.; Giese, C.; Krämer, R.; Ulber, R. An immobilized perylene diimide derivative for fucoidan purification from a crude brown algae extract. Process Biochem. 2018, 65, 233-238. [CrossRef]

28. Citkowska, A.; Szekalska, M.; Winnicka, K. Possibilities of fucoidan utilization in the development of pharmaceutical dosage forms. Mar. Drugs 2019, 17, 458. [CrossRef]

29. Li, S.; Li, J.; Zhi, Z.; Hu, Y.; Ge, J.; Ye, X.; Tian, D.; Linhardt, R.J.; Chen, S. 4-O-sulfation in sea cucumber fucodians contribute to reversing dyslipidiaemia caused by HFD. Int. J. Biol. Macromol. 2017, 99, 96-104. [CrossRef]

30. Fitton, J.H.; Stringer, D.S.; Park, A.Y.; Karpiniec, S.N. Therapies from fucoidan: new developments. Mar. Drugs 2019, 17, 571. [CrossRef]

31. Van Weelden, G.; Bobinski, M.; Okla, K.; van Weelden, W.J.; Romano, A.; Pijnenborg, J.M.A. Fucoidan structure and activity in relation to anti-cancer mechanisms. Mar. Drugs 2019, 17, 32. [CrossRef] [PubMed]

32. Wang, Y.; Xing, M.; Cao, Q.; Ji, A.; Liang, H.; Song, S. Biological activities of fucoidan and the factors mediating its therapeutic effects: A review of recent studies. Mar. Drugs 2019, 17, 183. [CrossRef] [PubMed]

33. Luthuli, S.; Wu, S.; Cheng, Y.; Zheng, X.; Wu, M.; Tong, H. Therapeutic effects of fucoidan: A review on recent studies. Mar. Drugs 2019, 17, 487. [CrossRef] [PubMed]

34. Chollet, L.; Saboural, P.; Chauvierre, C.; Villemin, J.N.; Letourneur, D.; Chaubet, F. Fucoidans in nanomedicine. Mar. Drugs 2016, 14, 145. [CrossRef] [PubMed]

35. Holtkamp, A.D.; Kelly, S.; Ulber, R.; Lang, S. Fucoidans and fucoidanases-focus on techniques for molecular structure elucidation and modification of marine polysaccharides. Appl. Microbiol. Biotechnol. 2009, 82, 1-11. [CrossRef] [PubMed]

36. Ustyuzhanina, N.E.; Bilan, M.I.; Ushakova, N.A.; Usov, A.I.; Kiselevskiy, M.V.; Nifantiev, N.E. Fucoidans: Pro- or antiangiogenic agents? Glycobiology 2014, 24, 1265-1274. [CrossRef]

37. Zhao, Y.; Zheng, Y.; Wang, J.; Ma, S.; Yu, Y.; White, W.L.; Yang, S.; Yang, F.; Lu, J. Fucoidan extracted from Undaria pinnatifida: Source for nutraceuticals/functional foods. Mar. Drugs 2018, 16, 321. [CrossRef]

38. Arumugam, P.; Arunkumar, K.; Sivakumar, L.; Murugan, M.; Murugan, K. Anticancer effect of fucoidan on cell proliferation, cell cycle progression, genetic damage and apoptotic cell death in HepG2 cancer cells. Toxicol. Rep. 2019, 6, 556-563.

39. Atashrazm, F.; Lowenthal, R.M.; Woods, G.M.; Holloway, A.F.; Dickinson, J.L. Fucoidan and cancer: A multifunctional molecule with anti-tumor potential. Mar. Drugs 2015, 13, 2327-2346. [CrossRef] 
40. Chen, L.-M.; Liu, P.-Y.; Chen, Y.-A.; Tseng, H.-Y.; Shen, P.-C.; Hwang, P.-A.; Hsu, H.-L. Oligo-fucoidan prevents IL-6 and CCL2 production and cooperates with p53 to suppress ATM signaling and tumor progression. Sci. Rep. 2017, 7, 11864. [CrossRef]

41. Zayed, A.; Muffler, K.; Hahn, T.; Rupp, S.; Finkelmeier, D.; Burger-Kentischer, A.; Ulber, R. Physicochemical and biological characterization of fucoidan from Fucus vesiculosus purified by dye affinity chromatography. Mar. Drugs 2016, 14, 79. [CrossRef] [PubMed]

42. Zhao, X.; Dong, S.; Wang, J.; Li, F.; Chen, A.; Li, B. A comparative study of antithrombotic and antiplatelet activities of different fucoidans from Laminaria japonica. Thromb. Res. 2012, 129, 771-778. [CrossRef] [PubMed]

43. Wang, W.; Wu, J.; Zhang, X.; Hao, C.; Zhao, X.; Jiao, G.; Shan, X.; Tai, W.; Yu, G. Inhibition of influenza A virus infection by fucoidan targeting viral neuraminidase and cellular EGFR pathway. Sci. Rep. 2017, 7, 40760. [CrossRef] [PubMed]

44. Prokofjeva, M.M.; Imbs, T.I.; Shevchenko, N.M.; Spirin, P.V.; Horn, S.; Fehse, B.; Zvyagintseva, T.N.; Prassolov, V.S. Fucoidans as potential inhibitors of HIV-1. Mar. Drugs 2013, 11, 3000-3014. [CrossRef] [PubMed]

45. Takahashi, H.; Kawaguchi, M.; Kitamura, K.; Narumiya, S.; Kawamura, M.; Tengan, I.; Nishimoto, S.; Hanamure, Y.; Majima, Y.; Tsubura, S.; et al. An exploratory study on the anti-inflammatory effects of fucoidan in relation to quality of life in advanced cancer patients. Integr. Cancer Ther. 2018, 17, 282-291. [CrossRef]

46. Park, H.Y.; Han, M.H.; Park, C.; Jin, C.-Y.; Kim, G.-Y.; Choi, I.-W.; Kim, N.D.; Nam, T.-J.; Kwon, T.K.; Choi, Y.H. Anti-inflammatory effects of fucoidan through inhibition of NF-kB, MAPK and Akt activation in lipopolysaccharide-induced $\mathrm{BV}_{2}$ microglia cells. Food Chem. Toxicol. 2011, 49, 1745-1752. [CrossRef]

47. Li, B.; Lu, F.; Wei, X.; Zhao, R. Fucoidan: Structure and bioactivity. Molecules 2008, 13, 1671-1695. [CrossRef]

48. Lu, J.; Shi, K.K.; Chen, S.; Wang, J.; Hassouna, A.; White, L.N.; Merien, F.; Xie, M.; Kong, Q.; Li, J.; et al. Fucoidan extracted from the New Zealand Undaria pinnatifida-Physicochemical comparison against five other fucoidans: Unique low molecular weight fraction bioactivity in breast cancer cell lines. Mar. Drugs 2018, 16, 461. [CrossRef]

49. Yoo, H.J.; You, D.-J.; Lee, K.-W. Characterization and immunomodulatory effects of high molecular weight fucoidan fraction from the sporophyll of Undaria pinnatifida in cyclophosphamide-induced immunosuppressed mice. Mar. Drugs 2019, 17, 447. [CrossRef]

50. Zayed, A.; Hahn, T.; Finkelmeier, D.; Burger-Kentischer, A.; Rupp, S.; Krämer, R.; Ulber, R. Phenomenological investigation of the cytotoxic activity of fucoidan isolated from Fucus vesiculosus. Process Biochem. 2019, 81, 182-187. [CrossRef]

51. Imbs, T.I.; Skriptsova, A.V.; Zvyagintseva, T.N. Antioxidant activity of fucose-containing sulfated polysaccharides obtained from Fucus evanescens by different extraction methods. J. Appl. Phycol. 2015, 27, 545-553. [CrossRef]

52. Hifney, A.F.; Fawzy, M.A.; Abdel-Gawad, K.M.; Gomaa, M. Industrial optimization of fucoidan extraction from Sargassum sp. and its potential antioxidant and emulsifying activities. Food Hydrocoll. 2016, 54, 77-88. [CrossRef]

53. Zayed, A.; Ulber, R. Fucoidan Production: Approval Key Challenges and Opportunities. Carbohydr. Polym. 2019, 211, 289-297. [CrossRef] [PubMed]

54. Fitton, J.H.; Stringer, D.N.; Karpiniec, S.S. Therapies from fucoidan: An update. Mar. Drugs 2015, 13, 5920-5946. [CrossRef]

55. Mak, W.; Wang, S.K.; Liu, T.; Hamid, N.; Li, Y.; Lu, J.; White, W.L. Anti-proliferation potential and content of fucoidan extracted from sporophyll of New Zealand Undaria pinnatifida. Front. Nutr. 2014, 1, 9. [CrossRef]

56. Chauvierre, C.; Aid-Launais, R.; Aerts, J.; Chaubet, F.; Maire, M.; Chollet, L.; Rolland, L.; Bonafe, R.; Rossi, S.; Bussi, S.; et al. Pharmaceutical development and safety evaluation of a GMP-grade fucoidan for molecular diagnosis of cardiovascular diseases. Mar. Drugs 2019, 17, 699. [CrossRef]

57. Torres, M.D.; Flórez-Fernández, N.; Simón-Vázquez, R.; Giménez-Abián, J.F.; Díaz, J.F.; González-Fernández, Á.; Domínguez, H. Fucoidans: The importance of processing on their anti-tumoral properties. Algal Res. 2020, 45, 101748. [CrossRef] 
58. Borazjani, N.J.; Tabarsa, M.; You, S.; Rezaei, M. Improved immunomodulatory and antioxidant properties of unrefined fucoidans from Sargassum angustifolium by hydrolysis. J. Food Sci. Technol. 2017, 54, 4016-4025. [CrossRef]

59. Koyanagi, S.; Tanigawa, N.; Nakagawa, H.; Soeda, S.; Shimeno, H. Oversulfation of fucoidan enhances its anti-angiogenic and antitumor activities. Biochem. Pharmacol. 2003, 65, 173-179. [CrossRef]

60. Ale, M.T.; Mikkelsen, J.D.; Meyer, A.S. Important determinants for fucoidan bioactivity: A critical review of structure-function relations and extraction methods for fucose-containing sulfated polysaccharides from brown seaweeds. Mar. Drugs 2011, 9, 2106-2130. [CrossRef]

61. Silchenko, A.S.; Rasin, A.B.; Kusaykin, M.I.; Malyarenko, O.S.; Shevchenko, N.M.; Zueva, A.O.; Kalinovsky, A.I.; Zvyagintseva, T.N.; Ermakova, S.P. Modification of native fucoidan from fucus evanescens by recombinant fucoidanase from marine bacteria Formosa algae. Carbohydr. Polym. 2018, 193, 189-195. [CrossRef] [PubMed]

62. Jiao, G.; Yu, G.; Zhang, J.; Ewart, H.S. Chemical structures and bioactivities of sulfated polysaccharides from marine algae. Mar. Drugs 2011, 9, 196-223. [CrossRef] [PubMed]

63. Suprunchuk, V.E. Low-molecular-weight fucoidan: Chemical modification, synthesis of its oligomeric fragments and mimetics. Carbohydr. Res. 2019, 485, 107806. [CrossRef] [PubMed]

64. Kardeby, C.; Fälker, K.; Haining, E.J.; Criel, M.; Lindkvist, M.; Barroso, R.; Påhlsson, P.; Ljungberg, L.U.; Tengdelius, M.; Rainger, G.E.; et al. Synthetic glycopolymers and natural fucoidans cause human platelet aggregation via PEAR1 and GPIb $\alpha$. Blood Adv. 2019, 3, 275-287. [CrossRef]

65. Kasai, A.; Arafuka, S.; Koshiba, N.; Takahashi, D.; Toshima, K. Systematic synthesis of low-molecular weight fucoidan derivatives and their effect on cancer cells. Org. Biomol. Chem. 2015, 13, 10556-10568. [CrossRef]

66. Rhein-Knudsen, N.; Ale, M.T.; Meyer, A.S. Seaweed hydrocolloid production: An update on enzyme assisted extraction and modification technologies. Mar. Drugs 2015, 13, 3340-3359. [CrossRef]

67. Buschmann, A.H.; Camus, C.; Infante, J.; Neori, A.; Israel, Á.; Hernández-González, M.C.; Pereda, S.V.; Gomez-Pinchetti, J.L.; Golberg, A.; Tadmor-Shalev, N.; et al. Seaweed production: Overview of the global state of exploitation, farming and emerging research activity. Eur. J. Phycol. 2017, 52, 391-406. [CrossRef]

68. Duarte, C.M.; Wu, J.; Xiao, X.; Bruhn, A.; Krause-Jensen, D. Can seaweed farming play a role in climate change mitigation and adaptation? Front. Mar. Sci. 2017, 4, 100.

69. Zayed, A.; Kovacheva, M.; Muffler, K.; Breiner, H.-W.; Stoeck, T.; Ulber, R. Induction and genetic identification of a callus-like growth developed in the brown alga Fucus vesiculosus. Eng. Life Sci. 2019, 19, 363-369. [CrossRef]

70. Cho, G.Y.; Rousseau, F.; de Reviers, B.; Boo, S.M. Phylogenetic relationships within the fucales (Phaeophyceae) assessed by the photosystem I coding psaa sequences. Phycologia 2006, 45, 512-519. [CrossRef]

71. Wahl, M.; Molis, M.; Hobday, A.J.; Dudgeon, S.; Neumann, R.; Steinberg, P.; Campbell, A.H.; Marzinelli, E.; Connell, S. The responses of brown macroalgae to environmental change from local to global scales: Direct versus ecologically mediated effects. Perspect. Phycol. 2015, 2, 11-29. [CrossRef]

72. Crous, P.W.; Wingfield, M.J.; Burgess, T.I.; Hardy, G.S.J.; Gené, J.; Guarro, J.; Baseia, I.G.; García, D.; Gusmão, L.F.P.; Souza-Motta, C.M.; et al. Fungal planet description sheets: 716-784. Persoonia 2018, 40, 240-393. [CrossRef] [PubMed]

73. Kumar, G.R.; Reddy, C.R.K.; Jha, B. Callus induction and thallus regeneration from callus of phycocolloid yielding seaweeds from the Indian coast. J. Appl. Phycol. 2007, 19, 15-25. [CrossRef]

74. Muhamad, S.N.S.; Ling, A.P.-K.; Wong, C.-L. Effect of plant growth regulators on direct regeneration and callus induction from Sargassum polycystum C. Agardh. J. Appl. Phycol. 2018, 30, 3299-3310. [CrossRef]

75. Avila-Peltroche, J.; Won, B.Y.; Cho, T.O. Protoplast isolation and regeneration from Hecatonema terminale (Ectocarpales, Phaeophyceae) using a simple mixture of commercial enzymes. J. Appl. Phycol. 2019, 31, 1873-1881. [CrossRef]

76. Luiten, E.E.; Akkerman, I.; Koulman, A.; Kamermans, P.; Reith, H.; Barbosa, M.J.; Sipkema, D.; Wijffels, R.H. Realizing the promises of marine biotechnology. Biomol. Eng. 2003, 20, 429-439. [CrossRef]

77. Baweja, P.; Sahoo, D.; García-Jiménez, P.; Robaina, R.R. Review: Seaweed tissue culture as applied to biotechnology: Problems, achievements and prospects. Phycol. Res. 2009, 57, 45-58. [CrossRef]

78. Huang, Y.M.; Rorrer, G.L. Cultivation of microplantlets derived from the marine red alga Agardhiella subulata in a stirred tank photobioreactor. Biotechnol. Prog. 2003, 19, 418-427. [CrossRef] 
79. Hahn, T.; Lang, S.; Ulber, R.; Muffler, K. Novel procedures for the extraction of fucoidan from brown algae. Process Biochem. 2012, 47, 1691-1698. [CrossRef]

80. Thinh, P.D.; Menshova, R.V.; Ermakova, S.P.; Anastyuk, S.D.; Ly, B.M.; Zvyagintseva, T.N. Structural characteristics and anticancer activity of fucoidan from the brown alga Sargassum mcclurei. Mar. Drugs 2013, 11, 1456-1476. [CrossRef]

81. De Reviers, B. Fucans and alginates without phenolic compounds. J. Appl. Phycol. 1989, 1, 75-76. [CrossRef]

82. Yang, W.N.; Chen, P.W.; Huang, C.Y. Compositional characteristics and in vitro evaluations of antioxidant and neuroprotective properties of crude extracts of fucoidan prepared from compressional puffing-pretreated Sargassum crassifolium. Mar. Drugs 2017, 15, 183. [CrossRef] [PubMed]

83. Zhang, R.; Zhang, X.; Tang, Y.; Mao, J. Composition, isolation, purification and biological activities of Sargassum fusiforme polysaccharides: A review. Carbohydr. Polym. 2020, 228, 115381. [CrossRef] [PubMed]

84. Spicer, S.E.; Adams, J.M.M.; Thomas, D.S.; Gallagher, J.A.; Winters, A.L. Novel rapid method for the characterisation of polymeric sugars from macroalgae. J. Appl. Phycol. 2017, 29, 1507-1513. [CrossRef]

85. Zou, P.; Lu, X.; Zhao, H.; Yuan, Y.; Meng, L.; Zhang, C.; Li, Y. Polysaccharides derived from the brown algae Lessonia nigrescens enhance salt stress tolerance to wheat seedlings by enhancing the antioxidant system and modulating intracellular ion concentration. Front. Plant Sci. 2019, 10, 48. [CrossRef]

86. Peng, J.; Yuan, J.-P.; Wu, C.-F.; Wang, J.-H. Fucoxanthin, a marine carotenoid present in brown seaweeds and diatoms: Metabolism and bioactivities relevant to human health. Mar. Drugs 2011, 9, 1806-1828. [CrossRef]

87. Da Costa, E.; Domingues, P.; Melo, T.; Coelho, E.; Pereira, R.; Calado, R.; Abreu, M.H.; Domingues, M.R. Lipidomic signatures reveal seasonal shifts on the relative abundance of high-valued lipids from the brown algae Fucus vesiculosus. Mar. Drugs 2019, 17, 335. [CrossRef]

88. Terasaki, M.; Hirose, A.; Narayan, B.; Baba, Y.; Kawagoe, C.; Yasui, H.; Saga, N.; Hosokawa, M.; Miyashita, K. Evaluation of recoverable functional lipid components of several brown seaweeds (Phaeophyta) from Japan with special reference to fucoxanthin and fucosterol contents. J. Phycol. 2009, 45, 974-980. [CrossRef]

89. Zhu, F. Interactions between cell wall polysaccharides and polyphenols. Crit. Rev. Food Sci. Nutr. 2018, 58, 1808-1831. [CrossRef]

90. Li, Y.; Fu, X.; Duan, D.; Liu, X.; Xu, J.; Gao, X. Extraction and Identification of Phlorotannins from the Brown Alga, Sargassum fusiforme (Harvey) Setchell. Mar. Drugs 2017, 15, 49. [CrossRef]

91. Bertoni, G. A key step in phlorotannin biosynthesis revealed. Plant Cell 2013, 25, 2770. [CrossRef] [PubMed]

92. Catarino, M.D.; Silva, A.M.S.; Cardoso, S.M. Fucaceae: A source of bioactive phlorotannins. Int. J. Mol. Sci. 2017, 18, 1327. [CrossRef] [PubMed]

93. Thomas, N.V.; Kim, S.K. Potential pharmacological applications of polyphenolic derivatives from marine brown algae. Environ. Toxicol. Pharmacol. 2011, 32, 325-335. [CrossRef] [PubMed]

94. Agregán, R.; Munekata, P.E.S.; Franco, D.; Carballo, J.; Barba, F.J.; Lorenzo, J.M. Antioxidant potential of extracts obtained from macro- (Ascophyllum nodosum, Fucus vesiculosus and Bifurcaria bifurcata) and micro-Algae (Chlorella vulgaris and Spirulina platensis) assisted by ultrasound. Medicines 2018, 5, 33.

95. Zhang, M.Y.; Guo, J.; Hu, X.M.; Zhao, S.Q.; Li, S.L.; Wang, J. An in vivo anti-tumor effect of eckol from marine brown algae by improving the immune response. Food Funct. 2019, 10, 4361-4371. [CrossRef]

96. Gall, E.A.; Lelchat, F.; Hupel, M.; Jégou, C.; Stiger-Pouvreau, V. Extraction and purification of phlorotannins from brown algae. In Natural Products from Marine Algae: Methods and Protocols; Stengel, D.B., Connan, S., Eds.; Springer: New York, NY, USA, 2015; pp. 131-143.

97. Brzonova, I.; Kozliak, E.I.; Andrianova, A.A.; LaVallie, A.; Kubátová, A.; Ji, Y. Production of lignin based insoluble polymers (anionic hydrogels) by C. versicolor. Sci. Rep. 2017, 7, 17507. [CrossRef]

98. Hahn, T.; Zayed, A.; Kovacheva, M.; Stadtmüller, R.; Lang, S.; Muffler, K.; Ulber, R. Dye affinity chromatography for fast and simple purification of fucoidan from marine brown algae. Eng. Life Sci. 2016, 16, 78-87. [CrossRef]

99. Chades, T.; Scully, S.M.; Ingvadottir, E.M.; Orlygsson, J. Fermentation of mannitol extracts from brown macro algae by Thermophilic clostridia. Front. Microbiol. 2018, 9, 1931. [CrossRef]

100. Balboa, E.M.; Rivas, S.; Moure, A.; Dominguez, H.; Parajo, J.C. Simultaneous extraction and depolymerization of fucoidan from Sargassum muticum in aqueous media. Mar. Drugs 2013, 11, 4612-4627. [CrossRef]

101. Descamps, V.; Colin, S.; Lahaye, M.; Jam, M.; Richard, C.; Potin, P.; Barbeyron, T.; Yvin, J.-C.; Kloareg, B. Isolation and culture of a marine bacterium degrading the sulfated fucans from marine brown algae. Mar. Biotechnol. 2006, 8, 27-39. [CrossRef] 
102. Abdella, A.A.; Ulber, R.; Zayed, A. Chitosan-toluidine blue beads for purification of fucoidans. Carbohydr. Polym. 2020, 231, 115686. [CrossRef] [PubMed]

103. Kadam, S.U.; Tiwari, B.K.; O'Donnell, C.P. Application of novel extraction technologies for bioactives from marine algae. J. Agric. Food Chem. 2013, 61, 4667-4675. [CrossRef]

104. Pozharitskaya, O.N.; Shikov, A.N.; Faustova, N.M.; Obluchinskaya, E.D.; Kosman, V.M.; Vuorela, H.; Makarov, V.G. Pharmacokinetic and tissue distribution of fucoidan from Fucus vesiculosus after oral administration to rats. Mar. Drugs 2018, 16, 132. [CrossRef] [PubMed]

105. Hadj Ammar, H.; Lajili, S.; Ben Said, R.; Le Cerf, D.; Bouraoui, A.; Majdoub, H. Physico-chemical characterization and pharmacological evaluation of sulfated polysaccharides from three species of Mediterranean brown algae of the genus Cystoseira. DARU J. Pharm. Sci. 2015, 23, 1. [CrossRef] [PubMed]

106. Zhang, Y.; Xu, M.; Hu, C.; Liu, A.; Chen, J.; Gu, C.; Zhang, X.; You, C.; Tong, H.; Wu, M.; et al. Sargassum fusiforme fucoidan SP2 extends the lifespan of Drosophila melanogaster by upregulating the Nrf2-mediated antioxidant signaling pathway. Oxidative Med. Cell. Longev. 2019, 2019, 8918914. [CrossRef]

107. Saepudin, E.; Sinurat, E.; Suryabrata, I.A. Depigmentation and characterization of fucoidan from brown seaweed Sargassum binderi Sonder. IOP Conference Series: Mater. Sci. Eng. 2018, 299, 012027. [CrossRef]

108. Patel, A.; Mikes, F.; Matsakas, L. An overview of current pretreatment methods used to improve lipid extraction from Oleaginous micro-organisms. Molecules 2018, 23, 1562. [CrossRef]

109. Huang, C.-Y.; Kuo, C.-H.; Chen, P.-W. Compressional-puffing pretreatment enhances neuroprotective effects of fucoidans from the brown seaweed Sargassum hemiphyllum on 6-hydroxydopamine-induced apoptosis in SH-SY5Y cells. Molecules 2017, 23, 78. [CrossRef]

110. Huang, C.-Y.; Wu, S.-J.; Yang, W.-N.; Kuan, A.-W.; Chen, C.-Y. Antioxidant activities of crude extracts of fucoidan extracted from Sargassum glaucescens by a compressional-puffing-hydrothermal extraction process. Food Chem. 2016, 197, 1121-1129. [CrossRef]

111. Kordjazi, M.; Shabanpour, B.; Zabihi, E.; Faramarzi, M.A.; Feizi, F.; Ahmadi Gavlighi, H.; Feghhi, M.A.; Hosseini, S.A. Sulfated polysaccharides purified from two species of Padina improve collagen and epidermis formation in the rat. Int. J. Mol. Cell. Med. 2013, 2, 156-163.

112. Cho, M.L.; Lee, B.-Y.; You, S.G. Relationship between oversulfation and conformation of low and high molecular weight fucoidans and evaluation of their in vitro anticancer activity. Molecules 2010, 16, 291-297. [CrossRef] [PubMed]

113. Oliveira, R.M.; Câmara, R.B.G.; Monte, J.F.S.; Viana, R.L.S.; Melo, K.R.T.; Queiroz, M.F.; Filgueira, L.G.A.; Oyama, L.M.; Rocha, H.A.O. Commercial fucoidans from Fucus vesiculosus can be grouped into antiadipogenic and adipogenic agents. Mar. Drugs 2018, 16, 193. [CrossRef] [PubMed]

114. Kusaykin, M.I.; Silchenko, A.S.; Zakharenko, A.M.; Zvyagintseva, T.N. Fucoidanases. Glycobiology 2015, 26, 3-12. [CrossRef] [PubMed]

115. Ale, M.T.; Meyer, A.S. Fucoidans from brown seaweeds: An update on structures, extraction techniques and use of enzymes as tools for structural elucidation. RSC Adv. 2013, 3, 8131-8141. [CrossRef]

116. Huang, C.-Y.; Kuo, C.-H.; Lee, C.-H. Antibacterial and antioxidant capacities and attenuation of lipid accumulation in 3T3-L1 adipocytes by low-molecular-weight fucoidans prepared from compressional-puffing-pretreated Sargassum crassifolium. Mar. Drugs 2018, 16, 24. [CrossRef] [PubMed]

117. Imbs, T.I.; Shevchenko, N.M.; Sukhoverkhov, S.V.; Semenova, T.L.; Skriptsova, A.V.; Zvyagintseva, T.N. Seasonal variations of the composition and structural characteristics of polysaccharides from the brown alga Costaria costata. Chem. Nat. Compd. 2009, 45, 786-791. [CrossRef]

118. Fidelis, G.P.; Silva, C.H.F.; Nobre, L.; Medeiros, V.P.; Rocha, H.A.O.; Costa, L.S. Antioxidant fucoidans obtained from tropical seaweed protect pre-osteoblastic cells from hydrogen peroxide-induced damage. Mar. Drugs 2019, 17, 506. [CrossRef]

119. Rohwer, K.; Neupane, S.; Bittkau, K.S.; Galarza Perez, M.; Dorschmann, P.; Roider, J.; Alban, S.; Klettner, A. Effects of Crude Fucus distichus Subspecies evanescens Fucoidan Extract on Retinal Pigment Epithelium Cells-Implications for Use in Age-Related Macular Degeneration. Mar. Drugs 2019, 17, 538. [CrossRef]

120. Rodriguez-Jasso, R.M.; Mussatto, S.I.; Pastrana, L.; Aguilar, C.N.; Teixeira, J.A. Microwave-assisted extraction of sulfated polysaccharides (fucoidan) from brown seaweed. Carbohydr. Polym. 2011, 86, 1137-1144. [CrossRef] 
121. Mussatto, S.I. Microwave-assisted extraction of fucoidan from marine algae. In Natural Products from Marine Algae: Methods and Protocols; Stengel, D.B., Connan, S., Eds.; Springer: New York, NY, USA, 2015; pp. $151-157$.

122. Alboofetileh, M.; Rezaei, M.; Tabarsa, M.; You, S. Ultrasound-assisted extraction of sulfated polysaccharide from Nizamuddinia zanardinii: Process optimization, structural characterization, and biological properties. J. Food Process Eng. 2019, 42, e12979. [CrossRef]

123. Garcia-Vaquero, M.; O'Doherty, J.V.; Tiwari, B.K.; Sweeney, T.; Rajauria, G. Enhancing the extraction of polysaccharides and antioxidants from macroalgae using sequential hydrothermal-assisted extraction followed by ultrasound and thermal technologies. Mar. Drugs 2019, 17, 457. [CrossRef] [PubMed]

124. Alboofetileh, M.; Rezaei, M.; Tabarsa, M.; You, S.; Mariatti, F.; Cravotto, G. Subcritical water extraction as an efficient technique to isolate biologically-active fucoidans from Nizamuddinia zanardinii. Int. J. Biol. Macromol. 2019, 128, 244-253. [CrossRef] [PubMed]

125. Qin, Y.; Yuan, Q.; Zhang, Y.; Li, J.; Zhu, X.; Zhao, L.; Wen, J.; Liu, J.; Zhao, L.; Zhao, J. Enzyme-assisted extraction optimization, characterization and antioxidant activity of polysaccharides from sea cucumber Phyllophorus proteus. Molecules 2018, 23, 590. [CrossRef]

126. Wijesinghe, W.A.; Jeon, Y.J. Enzyme-assistant extraction (EAE) of bioactive components: A useful approach for recovery of industrially important metabolites from seaweeds: A review. Fitoterapia 2012, 83, 6-12. [CrossRef]

127. Ahn, G.; Lee, W.; Kim, K.-N.; Lee, J.-H.; Heo, S.-J.; Kang, N.; Lee, S.-H.; Ahn, C.-B.; Jeon, Y.-J. A sulfated polysaccharide of Ecklonia cava inhibits the growth of colon cancer cells by inducing apoptosis. EXCLI J. 2015, 14, 294-306.

128. Badrinathan, S.; Shiju, T.M.; Sharon Christa, A.S.; Arya, R.; Pragasam, V. Purification and structural characterization of sulfated polysaccharide from Sargassum myriocystum and its efficacy in scavenging free radicals. Indian J. Pharm. Sci. 2012, 74, 549-555. [PubMed]

129. Liu, Y.; Huang, G. Extraction and derivatisation of active polysaccharides. J. Enzym. Inhib. Med. Chem. 2019, 34, 1690-1696. [CrossRef]

130. Zhao, D.; Xu, J.; Xu, X. Bioactivity of fucoidan extracted from Laminaria japonica using a novel procedure with high yield. Food Chem. 2018, 245, 911-918. [CrossRef]

131. Xing, R.; Liu, S.; Yu, H.; Chen, X.; Qin, Y.; Li, K.; Li, P. Extraction and separation of fucoidan from Laminaria japonica with chitosan as extractant. BioMed Res. Int. 2013, 2013, 193689. [CrossRef]

132. Ertani, A.; Francioso, O.; Tinti, A.; Schiavon, M.; Pizzeghello, D.; Nardi, S. Evaluation of seaweed extracts from Laminaria and Ascophyllum nodosum spp. as biostimulants in Zea mays L. using a combination of chemical, biochemical and morphological approaches. Front. Plant Sci. 2018, 9, 428. [CrossRef]

133. Lee, J.M.; Oh, S.Y.; Johnston, T.V.; Ku, S.; Ji, G.E. Biocatalysis of fucodian in Undaria pinnatifida sporophyll using Bifidobacterium longum RD47 for production of prebiotic fucosylated oligosaccharide. Mar. Drugs 2019, 17, 117. [CrossRef] [PubMed]

134. Somasundaram, S.N.; Shanmugam, S.; Subramanian, B.; Jaganathan, R. Cytotoxic effect of fucoidan extracted from Sargassum cinereum on colon cancer cell line HCT-15. Int. J. Biol. Macromol. 2016, 91. [CrossRef] [PubMed]

135. Ustyuzhanina, N.E.; Ushakova, N.A.; Zyuzina, K.A.; Bilan, M.I.; Elizarova, A.L.; Somonova, O.V.; Madzhuga, A.V.; Krylov, V.B.; Preobrazhenskaya, M.E.; Usov, A.I.; et al. Influence of fucoidans on hemostatic system. Mar. Drugs 2013, 11, 2444-2458. [CrossRef] [PubMed]

136. Saboural, P.; Chaubet, F.; Rouzet, F.; Al-Shoukr, F.; Azzouna, R.B.; Bouchemal, N.; Picton, L.; Louedec, L.; Maire, M.; Rolland, L.; et al. Purification of a low molecular weight fucoidan for SPECT molecular imaging of myocardial infarction. Mar. Drugs 2014, 12, 4851-4867. [CrossRef] [PubMed]

137. Garcia-Vaquero, M.; Rajauria, G.; O’Doherty, J.V.; Sweeney, T. Polysaccharides from macroalgae: Recent advances, innovative technologies and challenges in extraction and purification. Food Res. Int. 2017, 99, 1011-1020. [CrossRef]

138. Hahn, T.; Schulz, M.; Stadtmüller, R.; Zayed, A.; Muffler, K.; Lang, S.; Ulber, R. Cationic dye for the specific determination of sulfated polysaccharides. Anal. Lett. 2016, 49, 1948-1962. [CrossRef]

139. Lee, J.M.; Shin, Z.U.; Mavlonov, G.T.; Abdurakhmonov, I.Y.; Yi, T.-H. Solid-phase colorimetric method for the quantification of fucoidan. Appl. Biochem. Biotechnol. 2012, 168, 1019-1024. [CrossRef] [PubMed] 
140. Palanisamy, S.; Vinosha, M.; Manikandakrishnan, M.; Anjali, R.; Rajasekar, P.; Marudhupandi, T.; Manikandan, R.; Vaseeharan, B.; Prabhu, N.M. Investigation of antioxidant and anticancer potential of fucoidan from Sargassum polycystum. Int. J. Biol. Macromol. 2018, 116, 151-161. [CrossRef]

141. Cong, Q.; Chen, H.; Liao, W.; Xiao, F.; Wang, P.; Qin, Y.; Dong, Q.; Ding, K. Structural characterization and effect on anti-angiogenic activity of a fucoidan from Sargassum fusiforme. Carbohydr. Polym. 2016, 136, 899-907. [CrossRef]

142. Li, G.; Row, K.H. Magnetic molecularly imprinted polymers for recognition and enrichment of polysaccharides from seaweed. J. Sep. Sci. 2017, 40, 4765-4772. [CrossRef]

143. Guthrie, L.; Wolfson, S.; Kelly, L. The human gut chemical landscape predicts microbe-mediated biotransformation of foods and drugs. eLife 2019, 8, e42866. [CrossRef] [PubMed]

144. Wang, J.; Zhang, Q.; Zhang, Z.; Song, H.; Li, P. Potential antioxidant and anticoagulant capacity of low molecular weight fucoidan fractions extracted from Laminaria japonica. Int. J. Biol. Macromol. 2010, 46, 6-12. [CrossRef] [PubMed]

145. Lee, J.B.; Hayashi, K.; Hashimoto, M.; Nakano, T.; Hayashi, T. Novel antiviral fucoidan from sporophyll of Undaria pinnatifida (Mekabu). Chem. Pharm. Bull. 2004, 52, 1091-1094. [CrossRef] [PubMed]

146. Elizondo-Gonzalez, R.; Cruz-Suarez, L.E.; Ricque-Marie, D.; Mendoza-Gamboa, E.; Rodriguez-Padilla, C.; Trejo-Avila, L.M. In vitro characterization of the antiviral activity of fucoidan from Cladosiphon okamuranus against Newcastle disease virus. Virol. J. 2012, 9, 307. [CrossRef]

147. Jeong, J.-W.; Hwang, S.J.; Han, M.H.; Lee, D.-S.; Yoo, J.S.; Choi, I.-W.; Cha, H.-J.; Kim, S.; Kim, H.-S.; Kim, G.-Y.; et al. Fucoidan inhibits lipopolysaccharide-induced inflammatory responses in RAW 264.7 macrophages and zebrafish larvae. Mol. Cell. Toxicol. 2017, 13, 405-417. [CrossRef]

148. Klettner, A. Fucoidan as a potential therapeutic for major blinding diseases - a hypothesis. Mar. Drugs 2016, 14, 31. [CrossRef]

149. Barbosa, A.I.; Coutinho, A.J.; Costa Lima, S.A.; Reis, S. Marine polysaccharides in pharmaceutical applications: Fucoidan and chitosan as key players in the drug delivery match field. Mar. Drugs 2019, 17, 654. [CrossRef]

150. Wang, P.; Kankala, R.K.; Fan, J.; Long, R.; Liu, Y.; Wang, S. Poly-L-ornithine/fucoidan-coated calcium carbonate microparticles by layer-by-layer self-assembly technique for cancer theranostics. J. Mater. Sci. Mater. Med. 2018, 29, 68. [CrossRef]

151. Wang, X.; Shan, X.; Dun, Y.; Cai, C.; Hao, J.; Li, G.; Cui, K.; Yu, G. Anti-metabolic syndrome effects of fucoidan from Fucus vesiculosus via reactive oxygen species-mediated regulation of JNK, Akt, and AMPK signaling. Molecules 2019, 24, 3319. [CrossRef]

152. Sharma, G.; Kar, S.; Basu Ball, W.; Ghosh, K.; Das, P.K. The curative effect of fucoidan on visceral leishmaniasis is mediated by activation of MAP kinases through specific protein kinase C isoforms. Cell. Mol. Immunol. 2014, 11, 263-274. [CrossRef]

153. Varikuti, S.; Jha, B.K.; Volpedo, G.; Ryan, N.M.; Halsey, G.; Hamza, O.M.; McGwire, B.S.; Satoskar, A.R. Host-directed drug therapies for neglected tropical diseases caused by protozoan parasites. Front. Microbiol. 2018, 9, 2655. [CrossRef] [PubMed]

154. Jin, J.O.; Zhang, W.; Du, J.Y.; Wong, K.W.; Oda, T.; Yu, Q. Fucoidan can function as an adjuvant in vivo to enhance dendritic cell maturation and function and promote antigen-specific $\mathrm{T}$ cell immune responses. PLoS ONE 2014, 9, e99396. [CrossRef] [PubMed]

155. Tabarsa, M.; Dabaghian, E.H.; You, S.; Yelithao, K.; Cao, R.; Rezaei, M.; Alboofetileh, M.; Bita, S. The activation of NF- $\mathrm{kB}$ and MAPKs signaling pathways of RAW264.7 murine macrophages and natural killer cells by fucoidan from Nizamuddinia zanardinii. Int. J. Biol. Macromol. 2020, 148, 56-67. [CrossRef] [PubMed]

156. Wang, P.; Liu, Z.; Liu, X.; Teng, H.; Zhang, C.; Hou, L.; Zou, X. Anti-metastasis effect of fucoidan from Undaria pinnatifida sporophylls in mouse hepatocarcinoma Hca-F cells. PLoS ONE 2014, 9, e106071. [CrossRef] [PubMed]

157. Li, Y.; Zhao, W.; Wang, L.; Chen, Y.; Zhang, H.; Wang, T.; Yang, X.; Xing, F.; Yan, J.; Fang, X. Protective effects of fucoidan against hydrogen peroxide-induced oxidative damage in porcine intestinal epithelial cells. Animals 2019, 9, 1108. [CrossRef]

158. Chen, J.H.; Lim, J.D.; Sohn, E.H.; Choi, Y.S.; Han, E.T. Growth-inhibitory effect of a fucoidan from brown seaweed Undaria pinnatifida on Plasmodium parasites. Parasitol. Res. 2009, 104, 245-250. [CrossRef]

159. Jia, Y.; Sun, Y.; Weng, L.; Li, Y.; Zhang, Q.; Zhou, H.; Yang, B. Low molecular weight fucoidan protects renal tubular cells from injury induced by albumin overload. Sci. Rep. 2016, 6, 31759. [CrossRef] 
160. Chen, C.-H.; Sue, Y.-M.; Cheng, C.-Y.; Chen, Y.-C.; Liu, C.-T.; Hsu, Y.-H.; Hwang, P.-A.; Huang, N.-J.; Chen, T.-H. Oligo-fucoidan prevents renal tubulointerstitial fibrosis by inhibiting the CD44 signal pathway. Sci. Rep. 2017, 7, 40183. [CrossRef]

161. Abdel-Daim, M.M.; Abushouk, A.I.; Bahbah, E.I.; Bungău, S.G.; Alyousif, M.S.; Aleya, L.; Alkahtani, S. Fucoidan protects against subacute diazinon-induced oxidative damage in cardiac, hepatic, and renal tissues. Environ. Sci. Pollut. Res. 2020. Online ahead of print. [CrossRef]

162. Chen, M.-C.; Hsu, W.-L.; Hwang, P.-A.; Chou, T.-C. Low molecular weight fucoidan inhibits tumor angiogenesis through downregulation of HIF-1/VEGF signaling under hypoxia. Mar. Drugs 2015, 13, 4436-4451. [CrossRef]

163. Marinval, N.; Saboural, P.; Haddad, O.; Maire, M.; Bassand, K.; Geinguenaud, F.; Djaker, N.; Ben Akrout, K.; Lamy de la Chapelle, M.; Robert, R.; et al. Identification of a pro-angiogenic potential and cellular uptake mechanism of a LMW highly sulfated fraction of fucoidan from Ascophyllum nodosum. Mar. Drugs 2016, 14, 185. [CrossRef] [PubMed]

164. Cheng, Y.; Sibusiso, L.; Hou, L.; Jiang, H.; Chen, P.; Zhang, X.; Wu, M.; Tong, H. Sargassum fusiforme fucoidan modifies the gut microbiota during alleviation of streptozotocin-induced hyperglycemia in mice. Int. J. Biol. Macromol. 2019, 131, 1162-1170. [CrossRef] [PubMed]

165. Li, B.; Juenet, M.; Aid-Launais, R.; Maire, M.; Ollivier, V.; Letourneur, D.; Chauvierre, C. Development of polymer microcapsules functionalized with fucoidan to target p-selectin overexpressed in cardiovascular diseases. Adv. Healthc. Mater. 2017, 6, 1601200. [CrossRef] [PubMed]

166. Kim, Y.-I.; Oh, W.-S.; Song, P.H.; Yun, S.; Kwon, Y.-S.; Lee, Y.J.; Ku, S.-K.; Song, C.-H.; Oh, T.-H. Anti-photoaging effects of low molecular-weight fucoidan on ultraviolet B-irradiated mice. Mar. Drugs 2018, 16, 286. [CrossRef] [PubMed]

167. Kim, J.H.; Lee, J.-E.; Kim, K.H.; Kang, N.J. Beneficial effects of marine algae-derived carbohydrates for skin health. Mar. Drugs 2018, 16, 459. [CrossRef]

168. Fitton, J.H.; Dell'Acqua, G.; Gardiner, V.-A.; Karpiniec, S.S.; Stringer, D.N.; Davis, E. Topical benefits of two fucoidan-rich extracts from marine macroalgae. Cosmetics 2015, 2, 66-81. [CrossRef]

169. Song, Y.S.; Li, H.; Balcos, M.C.; Yun, H.-Y.; Baek, K.J.; Kwon, N.S.; Choi, H.-R.; Park, K.-C.; Kim, D.-S. Fucoidan promotes the reconstruction of skin equivalents. Korean J. Physiol. Pharmacol. 2014, 18, 327-331. [CrossRef]

170. Venkatesan, J.; Anil, S.; Kim, S.-K.; Shim, M.S. Seaweed polysaccharide-based nanoparticles: Preparation and applications for drug delivery. Polymers 2016, 8, 30. [CrossRef]

171. Pinheiro, A.C.; Bourbon, A.I.; Cerqueira, M.A.; Maricato, É.; Nunes, C.; Coimbra, M.A.; Vicente, A.A. Chitosan/fucoidan multilayer nanocapsules as a vehicle for controlled release of bioactive compounds. Carbohydr. Polym. 2015, 115, 1-9. [CrossRef]

172. Choi, D.G.; Venkatesan, J.; Shim, M.S. Selective anticancer therapy using pro-oxidant drug-loaded chitosan-fucoidan nanoparticles. Int. J. Mol. Sci. 2019, 20, 3220. [CrossRef]

173. Tsai, M.-h.; Chuang, C.-c.; Chen, C.-c.; Yen, H.-j.; Cheng, K.-m.; Chen, X.-a.; Shyu, H.-f.; Lee, C.-y.; Young, J.-j.; Kau, J.-h. Nanoparticles assembled from fucoidan and trimethylchitosan as anthrax vaccine adjuvant: In vitro and in vivo efficacy in comparison to CpG. Carbohydr. Polym. 2020, 236, 116041. [CrossRef]

174. Venkatesan, J.; Singh, S.K.; Anil, S.; Kim, S.-K.; Shim, M.S. Preparation, characterization and biological applications of biosynthesized silver nanoparticles with chitosan-fucoidan coating. Molecules 2018, 23, 1429. [CrossRef]

175. Hwang, P.-A.; Yan, M.-D.; Lin, H.-T.V.; Li, K.-L.; Lin, Y.-C. Toxicological evaluation of low molecular weight fucoidan in vitro and in vivo. Mar. Drugs 2016, 14, 121. [CrossRef] [PubMed]

176. Zhu, Z.; Zhu, B.; Ai, C.; Lu, J.; Wu, S.; Liu, Y.; Wang, L.; Yang, J.; Song, S.; Liu, X. Development and application of a HPLC-MS/MS method for quantitation of fucosylated chondroitin sulfate and fucoidan in sea cucumbers. Carbohydr. Res. 2018, 466, 11-17. [CrossRef] [PubMed]

177. Yu, L.; Xue, C.; Chang, Y.; Xu, X.; Ge, L.; Liu, G.; Wang, Y. Structure elucidation of fucoidan composed of a novel tetrafucose repeating unit from sea cucumber Thelenota ananas. Food Chem. 2014, 146, 113-119. [CrossRef]

178. Zhao, X.; Guo, F.; Hu, J.; Zhang, L.; Xue, C.; Zhang, Z.; Li, B. Antithrombotic activity of oral administered low molecular weight fucoidan from Laminaria Japonica. Thromb. Res. 2016, 144, 46-52. [CrossRef] 
179. Tsai, H.L.; Tai, C.J.; Huang, C.W.; Chang, F.R.; Wang, J.Y. Efficacy of low-molecular-weight fucoidan as a supplemental therapy in metastatic colorectal cancer patients: A double-blind randomized controlled trial. Mar. Drugs 2017, 15, 122. [CrossRef]

180. Jonsson, M.; Allahgholi, L.; Sardari, R.R.R.; Hreggviethsson, G.O.; Nordberg Karlsson, E. Extraction and modification of macroalgal polysaccharides for current and next-generation applications. Molecules 2020, 25, 930. [CrossRef] [PubMed]

181. Cumashi, A.; Ushakova, N.A.; Preobrazhenskaya, M.E.; D’Incecco, A.; Piccoli, A.; Totani, L.; Tinari, N.; Morozevich, G.E.; Berman, A.E.; Bilan, M.I.; et al. A comparative study of the anti-inflammatory, anticoagulant, antiangiogenic, and antiadhesive activities of nine different fucoidans from brown seaweeds. Glycobiology 2007, 17, 541-552. [CrossRef] [PubMed]

182. Silchenko, A.S.; Ustyuzhanina, N.E.; Kusaykin, M.I.; Krylov, V.B.; Shashkov, A.S.; Dmitrenok, A.S.; Usoltseva, R.V.; Zueva, A.O.; Nifantiev, N.E.; Zvyagintseva, T.N. Expression and biochemical characterization and substrate specificity of the fucoidanase from Formosa algae. Glycobiology 2017, 27, 254-263.

183. Silchenko, A.S.; Rasin, A.B.; Kusaykin, M.I.; Kalinovsky, A.I.; Miansong, Z.; Changheng, L.; Malyarenko, O.; Zueva, A.O.; Zvyagintseva, T.N.; Ermakova, S.P. Structure, enzymatic transformation, anticancer activity of fucoidan and sulphated fucooligosaccharides from Sargassum horneri. Carbohydr. Polym. 2017, 175, 654-660. [CrossRef] [PubMed]

184. Colin, S.; Deniaud, E.; Jam, M.; Descamps, V.; Chevolot, Y.; Kervarec, N.; Yvin, J.C.; Barbeyron, T.; Michel, G.; Kloareg, B. Cloning and biochemical characterization of the fucanase FcnA: Definition of a novel glycoside hydrolase family specific for sulfated fucans. Glycobiology 2006, 16, 1021-1032. [CrossRef] [PubMed]

185. Nagao, T.; Arai, Y.; Yamaoka, M.; Komatsu, F.; Yagi, H.; Suzuki, H.; Ohshiro, T. Identification and characterization of the fucoidanase gene from Luteolibacter algae H18. J. Biosci. Bioeng. 2018, 126, 567-572. [CrossRef] [PubMed]

186. Bakunina, I.; Nedashkovskaia, O.I.; Alekseeva, S.A.; Ivanova, E.P.; Romanenko, L.A.; Gorshkova, N.M.; Isakov, V.V.; Zviagintseva, T.N.; Mikhailov, V.V. Degradation of fucoidan by the marine proteobacterium Pseudoalteromonas citrea. Mikrobiologiia 2002, 71, 49-55. [PubMed]

187. Wu, Q.; Zhang, M.; Wu, K.; Liu, B.; Cai, J.; Pan, R. Purification and characteristics of fucoidanase obtained from Dendryphiella arenaria TM94. J. Appl. Phycol. 2011, 23, 197-203. [CrossRef]

188. Sakai, T.; Kimura, H.; Kojima, K.; Shimanaka, K.; Ikai, K.; Kato, I. Marine bacterial sulfated fucoglucuronomannan (SFGM) lyase digests brown algal SFGM into trisaccharides. Mar. Biotechnol. 2003, 5, 70-78. [CrossRef]

189. Sakai, T.; Ishizuka, K.; Shimanaka, K.; Ikai, K.; Kato, I. Structures of oligosaccharides derived from Cladosiphon okamuranus fucoidan by digestion with marine bacterial enzymes. Mar. Biotechnol. 2003, 5, 536-544.

190. Ohshiro, T.; Ohmoto, Y.; Ono, Y.; Ohkita, R.; Miki, Y.; Kawamoto, H.; Izumi, Y. Isolation and characterization of a novel fucoidan-degrading microorganism. Biosci. Biotechnol. Biochem. 2010, 74, 1729-1732. [CrossRef]

191. Bilan, M.I.; Kusaykin, M.I.; Grachev, A.A.; Tsvetkova, E.A.; Zvyagintseva, T.N.; Nifantiev, N.E.; Usov, A.I. Effect of enzyme preparation from the marine mollusk Littorina kurila on fucoidan from the brown alga Fucus distichus. Biochemistry 2005, 70, 1321-1326. [CrossRef]

192. Kim, W.J.; Kim, S.M.; Lee, Y.H.; Kim, H.G.; Kim, H.K.; Moon, S.H.; Suh, H.H.; Jang, K.H.; Park, Y.I. Isolation and characterization of marine bacterial strain degrading fucoidan from korean Undaria pinnatifida Sporophylls. J. Microbiol. Biotechnol. 2008, 18, 616-623.

193. Kim, W.J.; Park, J.W.; Park, J.K.; Choi, D.J.; Park, Y.I. Purification and characterization of a fucoidanase (FNase S) from a marine bacterium Sphingomonas paucimobilis PF-1. Mar. Drugs 2015, 13, 4398-4417. [CrossRef]

194. Kitamura, K.; Matsuo, M.; Tsuneo, Y. Enzymic degradation of fucoidan by fucoidanase from the hepatopancreas of Patinopecten yessoensis. Biosci. Biotechnol. Biochem. 1992, 56, 490-494. [CrossRef]

195. Daniel, R.; Berteau, O.; Chevolot, L.; Varenne, A.; Gareil, P.; Goasdoue, N. Regioselective desulfation of sulfated l-fucopyranoside by a new sulfoesterase from the marine mollusk Pecten maximus. Eur. J. Biochem. 2001, 268, 5617-5626. [CrossRef]

196. Berteau, O.; McCort, I.; Goasdoué, N.; Tissot, B.; Daniel, R. Characterization of a new alpha-L-fucosidase isolated from the marine mollusk Pecten maximus that catalyzes the hydrolysis of alpha-L-fucose from algal fucoidan (Ascophyllum nodosum). Glycobiology 2002, 12, 273-282. [CrossRef] 
197. Dong, S.; Chang, Y.; Shen, J.; Xue, C.; Chen, F. Purification, expression and characterization of a novel $\alpha$-l-fucosidase from a marine bacteria Wenyingzhuangia fucanilytica. Protein Expr. Purif. 2017, 129, 9-17. [CrossRef]

198. Tanaka, R.; Mizutani, Y.; Shibata, T.; Miyake, H.; Iehata, S.; Mori, T.; Kuroda, K.; Ueda, M. Genome sequence of Formosa haliotis strain MA1, a brown alga-degrading bacterium isolated from the gut of Abalone Haliotis gigantea. Genome Announc. 2016, 4, e01312-16. [CrossRef]

199. Chen, F.; Chang, Y.; Dong, S.; Xue, C. Wenyingzhuangia fucanilytica sp. nov., a sulfated fucan utilizing bacterium isolated from shallow coastal seawater. Int. J. Syst. Evol. Microbiol. 2016, 66, 3270-3275. [CrossRef]

200. Li, J.; Cheng, Y.; Wang, D.; Li, J.; Wang, Y.; Han, W.; Li, F. Draft genome sequence of the polysaccharide-degrading marine bacterium Pseudoalteromonas sp. Strain A601. Genome Announc. 2017, 5, e00590-17. [CrossRef]

201. Cao, H.T.T.; Mikkelsen, M.D.; Lezyk, M.J.; Bui, L.M.; Tran, V.T.T.; Silchenko, A.S.; Kusaykin, M.I.; Pham, T.D.; Truong, B.H.; Holck, J.; et al. Novel enzyme actions for sulphated galactofucan depolymerisation and a new engineering strategy for molecular stabilisation of fucoidan degrading enzymes. Mar. Drugs 2018, 16, 422. [CrossRef]

202. Tran, P.H.L.; Duan, W.; Tran, T.T.D. Fucoidan-based nanostructures: A focus on its combination with chitosan and the surface functionalization of metallic nanoparticles for drug delivery. Int. J. Pharm. 2019, 575, 118956. [CrossRef]

203. Jesumani, V.; Du, H.; Pei, P.; Aslam, M.; Huang, N. Comparative study on skin protection activity of polyphenol-rich extract and polysaccharide-rich extract from Sargassum vachellianum. PLoS ONE 2020, 15, e0227308. [CrossRef] [PubMed]

204. Brunt, E.G.; Burgess, J.G. The promise of marine molecules as cosmetic active ingredients. Int. J. Cosmet. Sci. 2018, 40, 1-15. [CrossRef] [PubMed]

205. Ferreira, R.M.; Ramalho Ribeiro, A.; Patinha, C.; Silva, A.M.S.; Cardoso, S.M.; Costa, R. Water extraction kinetics of bioactive compounds of Fucus vesiculosus. Molecules 2019, 24, 3408. [CrossRef] [PubMed]

206. Michel, G.; Tonon, T.; Scornet, D.; Cock, J.M.; Kloareg, B. The cell wall polysaccharide metabolism of the brown alga Ectocarpus siliculosus. Insights into the evolution of extracellular matrix polysaccharides in eukaryotes. New Phytol. 2010, 188, 82-97. [CrossRef]

207. Chi, S.; Liu, T.; Wang, X.; Wang, R.; Wang, S.; Wang, G.; Shan, G.; Liu, C. Functional genomics analysis reveals the biosynthesis pathways of important cellular components (alginate and fucoidan) of Saccharina. Curr. Genet. 2018, 64, 259-273. [CrossRef] [PubMed]

208. Shao, Z.; Zhang, P.; Lu, C.; Li, S.; Chen, Z.; Wang, X.; Duan, D. Transcriptome sequencing of Saccharina Japonica Sporophytes during whole developmental periods reveals regulatory networks underlying alginate and mannitol biosynthesis. BMC Genom. 2019, 20, 975. [CrossRef] 